\title{
Re-processing the Hipparcos Transit Data and Intermediate Astrometric Data of spectroscopic binaries
}

\author{
I. Ba, CH and Tc-poor S stars \\ D. Pourbaix ${ }^{\star \star \star \star \star \star}$ and A. Jorissen ${ }^{\dagger}$
}

Institut d'Astronomie et d'Astrophysique, Université Libre de Bruxelles, CP. 226, Boulevard du Triomphe, B-1050 Bruxelles, Belgium

Received November 4, 1999; accepted May 4, 2000

\begin{abstract}
Only 235 entries were processed as astrometric binaries with orbits in the Hipparcos and Tycho Catalogue (ESA 1997). However, the Intermediate Astrometric Data (IAD) and Transit Data (TD) made available by ESA make it possible to re-process the stars that turned out to be spectroscopic binaries after the completion of the Catalogue. This paper illustrates how TD and IAD may be used in conjunction with the orbital parameters of spectroscopic binaries to derive astrometric parameters. The five astrometric and four orbital parameters (not already known from the spectroscopic orbit) are derived by minimizing an objective function $\left(\chi^{2}\right)$ with an algorithm of global optimization. This code has been applied to 81 systems for which spectroscopic orbits became available recently and that belong to various families of chemicallypeculiar red giants (namely, dwarf barium stars, strong and mild barium stars, CH stars, and Tc-poor S stars). Among these 81 systems, 23 yield reliable astrometric orbits. These 23 systems make it possible to evaluate on real data the so-called "cosmic error" described by Wielen et al. (1997), namely the fact that an unrecognized orbital motion introduces a systematic error on the proper motion. Comparison of the proper motion from the Hipparcos catalogue with that re-derived in the present work indicates that the former are indeed far off the present value for binaries with periods in the range 3 to $\sim 8$ years. Hipparcos parallaxes of unrecognized spectroscopic binaries turn out to be reliable, except for systems with periods close to 1 year, as expected. Finally, we show that, even when a complete orbital revolution was observed by Hipparcos, the inclination is unfortunately seldom precise.
\end{abstract}

Send offprint requests to: pourbaix@astro.ulb.ac.be

* Based on observations from the Hipparcos astrometric satellite operated by the European Space Agency (ESA 1997).

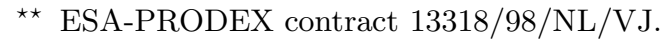

$\star \star \star$ Scientific Collaborator, F.N.R.S., Belgium.

$\dagger$ Research Associate, F.N.R.S., Belgium.
Key words: methods: data analysis - astrometry binaries: spectroscopic — stars: late-type — stars: fundamental parameters

\section{Introduction}

The chemical anomalies observed in several classes of latetype stars are due to mass transfer across a binary system. This scenario, first suggested by McClure (1983), holds for dwarf and giant barium stars (Pop. I G and $\mathrm{K}$ stars with overabundance of carbon and heavy elements like $\mathrm{Sr}$ and Ba produced by the s-process of nucleosynthesis), CH stars (the Pop. II analogs of Ba stars), and extrinsic $\mathrm{S}$ stars (late-type giants characterized by $\mathrm{ZrO}$ bands and no Tc lines, an element with no stable isotopes). The set of spectroscopic orbits available for those chemicallypeculiar red stars (CPRS) has been considerably enlarged thanks to a decade-long radial-velocity monitoring. Such orbits are now available for 63 giant barium stars and 19 extrinsic S stars (Jorissen et al. 1998), 21 dwarf barium and subgiant CH stars (McClure 1997; North, priv. comm.) and $8 \mathrm{CH}$ stars (McClure \& Woodsworth 1990). Most of them were not available to the Hipparcos reduction consortia. The astrometric parameters (parallaxes and proper motions) published in the Hipparcos catalogue (ESA 1997) were therefore derived from a single star solution (the so-called "5-parameter model"). Since many of these CPRS have orbital periods in the range $1-5 \mathrm{yr}$, their unaccounted orbital motion may seriously confuse the parallax and proper motion as determined by the Hipparcos consortia. Thus, unrecognized orbital motions might introduce a so-called "cosmic error" (of a systematic nature) in the proper motion (Wielen 1997; Wielen et al. 1999). Parallaxes may also possibly be in 
error, especially for binaries with orbital periods close to $1 \mathrm{yr}$.

The conclusions of previous studies inferring the kinematics and absolute magnitudes of CPRS (Bergeat \& Knapik 1997; Mennessier et al. 1997; Van Eck et al. 1998) from the data of the Hipparcos catalogue may possibly be affected by these systematic errors. It is therefore of importance to re-evaluate the astrometric parameters of CPRS with a model correctly accounting for the orbital motion (the so-called "12-parameter model", reducing to 9 parameters if the spectroscopic orbital parameters $P, T$ and $e$ are fixed), and to compare these values with those from the Hipparcos catalogue to identify the stars most affected by these systematic errors.

This comparison should allow to assess the reliability of the kinematic properties and absolute magnitudes of CPRS formerly derived from the Hipparcos-catalogue values. As a by-product, the astrometric orbit has been obtained in many cases, leading to an estimate of the masses through the ratio $M_{2} /\left[1+\left(M_{1} / M_{2}\right)\right]^{2} \equiv f(M) / \sin ^{3} i$ (derived by combining the orbital inclination from the astrometric orbit with the mass function $f(M)$ from the spectroscopic orbit when only one spectrum is observable).

From the operational point of view, the numerical re-processing of the raw Hipparcos data (Intermediate Astrometric Data or Transit Data; see Sect. 2.1) presented in this paper is innovative on the following points: (i) a global optimization technique is used to minimize the objective function in the 12-parameter (or, for spectroscopic binaries, 9-parameter) space, and (ii) a change of independent variable allows to derive always positive parallaxes, contrarily to the situation prevailing in the Hipparcos catalogue. The tool developed here for CPRS may in the future be applied to any other binary star to re-process the Hipparcos data when new orbits appear. The second paper of this series will be devoted to the re-processing of spectroscopic binaries involving a giant component (Boffin et al. 1993). Another is devoted to Procyon (Girard et al. 2000). We encourage interested readers to communicate to the authors other spectroscopic binaries whose Hipparcos data would need to be reprocessed.

\section{Numerical methods}

In order to allow some re-processing of the Hipparcos data, the Hipparcos consortia provided the users with two kinds of data: the Transit Data (TD) and the Intermediate Astrometric Data (IAD). TD (Quist \& Lindegren 1999) are a by-product of the analysis performed by the NDAC consortium, and merge astrometric and photometric data. They basically provide the signal as modulated by the grid in front of the detector. They are therefore especially well suited to the analysis of visual double or multiple systems, since the separation, position angle and magnitudes of the various components may in principle be extracted from the TD. For that reason, TD are not provided for all Hipparcos entries, but only for those stars known or suspected of being double or multiple systems. IAD are lower level data which provide astrometric information only (the star's abscissa along a reference great circle, and the pole of that circle on the sky; van Leeuwen \& Evans 1998), but for every Hipparcos entry. In the following, we describe how IAD and TD (when available) have been used to derive the astrometric parameters of the CPRS that have a known spectroscopic orbit. When TD are available, the astrometric parameters may be derived independently from the two data sets, and allow an interesting internal consistency check. In both cases, the method proceeds along the following steps:

- definition of an objective function to minimize;

- computation of the objective function from the astrometric parameters;

- global minimization of the objective function with the simulated annealing algorithm;

- local minimization of the objective function (with a quasi-Newton method);

- comparison of the values of the objective function for the Hipparcos 5-parameter model and for our 9parameter model, and application of an F-test to evaluate whether the 9-parameter model is significantly better than the Hipparcos solution;

- check of the astrophysical consistency of the 9parameter solution from some a priori knowledge of the properties of the system (i.e., component masses).

These steps are discussed in turn in the remaining of this section, first for IAD, and then for TD.

\subsection{Intermediate Astrometric Data}

\subsubsection{Objective function}

The first step in any data-fitting problem is to set up an objective function in order to compare different solutions (corresponding to different values of the model parameters). This function is usually constructed in such a way that its lowest value corresponds to the best solution. The problem then reduces to minimizing that function. The IAD already include some corrections (e.g. aberration and satellite-attitude corrections) and they are no longer stricto sensu raw data. They provide the abscissa residuals along a reference great circle for the Hipparcos 5parameter solution (i.e. $\alpha_{0}, \delta_{0}, \varpi, \mu_{\alpha}, \mu_{\delta}$, respectively: the position in right ascension and declination in the equinox 2000.0 at the epoch 1991.25, the parallax, the proper motions in right ascension and declination; following the practice of the Hipparcos catalogue, $\alpha_{0}^{*}=\alpha_{0} \cos \delta$ and $\mu_{\alpha}^{*}=\mu_{\alpha} \cos \delta$ will be used instead of $\alpha_{0}$ and $\mu_{\alpha}$ ). By slightly modifying these 5 parameters and by adding 7 orbital parameters (Binnendijk 1960), the aim is to further reduce the abscissa residuals $\Delta v$ below the values obtained 
from the Hipparcos 5-parameter model. In the case of the Hipparcos Intermediate Astrometric Data, the objective function is the $\chi^{2}$ expressed by Eq. (17.12) of Vol. 3 in Hipparcos:

$\chi^{2}=\left(\Delta v-\sum_{k=1}^{M} \frac{\partial v}{\partial p_{k}} \Delta p_{k}\right)^{t} V^{-1}\left(\Delta v-\sum_{k=1}^{N} \frac{\partial v}{\partial p_{k}} \Delta p_{k}\right)$

where the superscript $t$ means transposed, $\Delta v$ are the abscissa residuals provided by the IAD file and corresponding to the Hipparcos 5-parameter solution, and $\frac{\partial v}{\partial p_{k}}$ is the partial derivative of the abscissa with respect to the $k$-th parameter expressing how the abscissa residual varies when a correction $\Delta p_{k}$ is applied to the value of the $k$-th parameter with respect to the Hipparcos solution. $M$ is the number of parameters retained in the solution, and $V^{-1}$ is the inverse of the covariance matrix of the observations given by:

$V=\left(\begin{array}{cccc}V_{1} & 0 & \cdots & 0 \\ 0 & V_{2} & \ddots & \vdots \\ \vdots & \ddots & \ddots & 0 \\ 0 & \cdots & 0 & V_{n}\end{array}\right)$

with

$V_{j}=\left(\begin{array}{cc}\sigma_{F_{j}}^{2} & \rho \sigma_{F_{j}} \sigma_{N_{j}} \\ \rho \sigma_{F_{j}} \sigma_{N_{j}} & \sigma_{N_{j}}^{2}\end{array}\right)$.

If an observation $j$ was processed by the two consortia (FAST and NDAC), the residuals obtained by the two reduction consortia are correlated and $V_{j}$ is the $2 \times 2$ variance-covariance matrix for measurement $j$. On the other hand, when only one consortium processed observation $j, V_{j}$ reduces to one number ${ }^{1}$ (the estimated uncertainty $\sigma_{j}$ of the measurement). $\Delta v$, together with $\frac{\partial v}{\partial p_{k}}$ $\left(k=1\right.$ to 5, with $p_{1} \equiv \alpha_{0}^{*}, p_{2} \equiv \delta, p_{3} \equiv \varpi, p_{4} \equiv \mu_{\alpha^{*}}, p_{5} \equiv$ $\left.\mu_{\delta}\right)$, the original astrometric parameters as well as $\rho, \sigma_{F_{j}}$ and $\sigma_{N_{j}}$ are given in the IAD file. In order to evaluate $\chi^{2}$ (Eq. 1) for an orbital model, expressions for the partial derivatives of $v$ with respect to the orbital parameters are required. They can be expressed as a function of the partial derivatives of $v$ with respect to $\alpha_{0}^{*}$ and $\delta_{0}$ as follows (Hipparcos catalogue, Vol. 3, Eq. (17.15)):

$\frac{\partial v}{\partial o}=\frac{\partial v}{\partial \alpha_{0}^{*}} \frac{\partial \xi}{\partial o}+\frac{\partial v}{\partial \delta_{0}} \frac{\partial \eta}{\partial o}$

where $o$ is any orbital parameter and

$$
\xi=\alpha_{0}^{*}+\mu_{\alpha^{*}}\left(t-t_{0}\right)+R P_{\alpha} \varpi+y
$$$$
\eta=\delta_{0}+\mu_{\delta}\left(t-t_{0}\right)+R P_{\delta} \varpi+x \text {. }
$$

\footnotetext{
${ }^{1}$ In order to have a unique expression for $\chi^{2}$ regardless of the dataset available (FAST, NDAC or both), we always write $V_{j}$ as a 2 by 2 matrix. When, for whatever reason, a measurement from only one consortium is used, that observation is duplicated and its weight divided by two $\left(V_{j}=\operatorname{diag}\left(2 \sigma_{j}^{2}, 2 \sigma_{j}^{2}\right)\right)$. There is no change on the actual value of $\chi^{2}$ but its expression is easier (though not faster!) to evaluate.
}

In the above expression, $\xi$ and $\eta$ represent the Cartesian coordinates of the observed component on the plane tangent to the sky at the position $\left(\alpha_{0}, \delta_{0}\right)$. They combine the displacements due to the proper motion, the orbital motion and the parallax. $R$ is the radius vector of the Earth's orbit in A.U. at time $t$. $P_{\alpha}$ and $P_{\delta}$, the parallax factors, are given by Binnendijk (1960):

$$
\begin{aligned}
P_{\alpha}= & \cos \epsilon \cos \alpha \sin \odot-\sin \alpha \sin \odot \\
P_{\delta}= & (\sin \epsilon \cos \delta-\cos \epsilon \sin \alpha \sin \delta) \sin \odot \\
& -\cos \alpha \sin \delta \cos \odot
\end{aligned}
$$

where $\odot$ and $\epsilon$ are respectively the longitude of the Sun and the obliquity of the ecliptic, both at time $t$. The variables $x$ and $y$ describe the apparent orbit (i.e., projected on the plane orthogonal to the line of sight) of the observable component around the center of mass of the system. They are usually expressed in terms of the ThieleInnes constants $A, B, F, G$ of the photocentric orbit as (Heintz 1978)

$$
\begin{aligned}
& x=A X+F Y \\
& y=B X+G Y \\
& \text { with } \\
& X=\cos E-e \\
& Y=\sqrt{1-e^{2}} \sin E,
\end{aligned}
$$

where $E$ is the eccentric anomaly and $(X, Y)$ are the coordinates in the true orbit. It should be noted that $(x, y)$ and $(X, Y)$ are referred to a Cartesian system with $x$ pointing towards the North (Heintz 1978), contrary to $(\xi, \eta)$ where $\xi$ points towards increasing right ascensions. For all the systems considered in the present paper, the magnitude difference between the two components is larger than the Hipparcos detection threshold (since the companion to CPRS is a cool white dwarf; Sect. 5 and Jorissen et al. 1998). Thus, one can safely assume that there is no light coming from the secondary. Hence, the orbit of the photocenter of the primary component is the same as the absolute orbit of the primary around the center of mass of the system.

At this point, it is important to realize that the Hipparcos 5-parameter solution, that is used as a starting point for the new 12-parameter solution, is in fact equivalent to a 12-parameter solution where the semi-major axis of the orbit $\left(a_{0}\right)$ is null. It is therefore enough to consider in Eq. (1) the correction term relative to the semi-major axis of the orbit (and in fact $\Delta a_{0}=a_{0}$, since the initial value of $a_{0}$ is null). The other orbital parameters $i, \omega, \Omega, e, P$ and $T$ enter Eq. (1) only through the partial derivatives $\frac{\partial \xi}{\partial a_{0}}$ and $\frac{\partial \eta}{\partial a_{0}}$ [equal, respectively, to $\frac{\partial y}{\partial a_{0}}$ and $\frac{\partial x}{\partial a_{0}}$ according to Eq. (3)] entering $\frac{\partial v}{\partial a_{0}}$. These other orbital parameters do not require explicit correction terms in Eq. (1) (and their starting values would be ill-defined anyway). Hence, the orbital solution is the one minimizing

$\chi^{2}=\Xi^{t} V^{-1} \Xi$ 
where

$\Xi=\Delta v-\sum_{k=1}^{5} \frac{\partial v}{\partial p_{k}} \Delta p_{k}-\left(\frac{\partial v}{\partial p_{1}} \frac{\partial \xi}{\partial a_{0}}+\frac{\partial v}{\partial p_{2}} \frac{\partial \eta}{\partial a_{0}}\right) a_{0}$

is a vector of dimension $N(N$ is the number of observations), and $\xi$ and $\eta$ are functions of $a_{0}, i, \omega, \Omega, e, P$ and $T$, and $\frac{\partial \xi}{\partial a_{0}} \equiv \frac{\partial y}{\partial a_{0}}, \frac{\partial \eta}{\partial a_{0}} \equiv \frac{\partial x}{\partial a_{0}}$. Thus, the 12 parameters enter the evaluation of $\chi^{2}$ although there are only six correction terms subtracted from $\Delta v$.

Our experience has shown that, except for very special cases (i.e., parallaxes and semi-major axes larger than about 20 mas, orbital periods significantly different from 1 year but smaller than 3 years; one example is HIP 50805 in Table 1), astrometric orbits could not be derived from the IAD without an a priori knowledge of some of the orbital elements, for instance the spectroscopic ones $(e, P$ and $T$ ). However, it appeared that fixing $\omega$ at the value derived from the spectroscopic orbit often led to orbital inclinations $i$ unrealistically close to zero for spectroscopic binaries with radial velocity variations. Leaving the parameter $\omega$ free removes this difficulty, and offers moreover a way to check the consistency of the astrometric solution, since the astrometric $\omega$ should be consistent with its spectroscopic value.

As far as outliers are concerned, we almost always keep the same data set as that used by FAST and/or NDAC (Vol. 3, Sect. 17.6; Hipparcos). IAD that were not considered by FAST or NDAC (i.e., with the IA2 field set to "f" or " $\mathrm{f}$ ") were thus not included in our re-processing either. In a few cases, we noticed that because of the orbital contribution, some observations yield residuals larger than $3 \sigma$ of the residuals. In these cases, these observations were removed and the fit re-iterated. We never had to iterate more than twice to remove all outliers.

\subsubsection{Forcing parallaxes to be positive}

One of the five astrometric parameters entering $\chi^{2}$ in Eq. (1) is the parallax $\varpi$. With no other prescriptions as those described in Sect. 2.1.1, the minimization process may very well end up with a negative parallax. Indeed, negative parallaxes are not rare in the Hipparcos and Tycho catalogues.

Parallaxes cannot only be seen as inverse distances (which are defined positive) but also as the semi-major axis of the parallactic ellipse (see Eq. (3)). The direction of motion along the parallactic ellipse is of course imposed by the annual revolution of the Earth around the Sun, regardless of the actual dimension of the parallactic ellipse or of the observational uncertainties. In that sense, the parallactic ellipse is oriented, and negative parallaxes can be seen as corresponding to a parallactic ellipse covered in the wrong direction. That constraint being of physical nature, one should seek to fulfill it. In this section, we present a method which always delivers positive parallaxes. This method is especially useful for stars like those
Mira variables or carbon stars that came out with large negative parallaxes in the Hipparcos Catalogue. In those cases, forcing the parallax to be positive has a strong impact on the derived proper motion (Pourbaix et al., in preparation), which may be supposed to be better determined with a physically- sound model yielding positive parallaxes. However, the major drawback of the method is that the errors on the parallax do no longer follow a normal distribution. Therefore, the use of the parallaxes provided by this paper for e.g., luminosity calibrations should be done with care to avoid biases.

In order to force the parallax to be positive - and at the same time avoiding the difficulties inherent to any constrained minimization techniques - one may replace the constrained variable (the parallax $\varpi$ ) by an unconstrained one $^{2}$. An appropriate choice appears to be

$\varpi^{\prime}=\log \varpi$

which is $C^{\infty}$ between $] 0,+\infty\left[\right.$ and $\mathbb{R}$. The variable $\varpi^{\prime}$ is in fact equivalent to the distance modulus $m-M$ since

$m-M=-5-5 \varpi^{\prime}$.

The variable $\varpi^{\prime}$ is thus used in the minimization process instead of $\varpi$ as one of the $p_{k}$ parameters entering Eq. (1) [with $\left.\frac{\partial v}{\partial \varpi^{\prime}}=\frac{\partial v}{\partial \varpi} \varpi \ln 10\right]$. At the end of the minimization, Eq. (7) is reversed and the parallax $\varpi$ is derived from $\varpi^{\prime}$. For any real number $\varpi^{\prime}, \varpi$ necessarily lies in between 0 and $\infty$. Such a substitution is legitimate, since the parallax is not a directly measured quantity, but rather one among many parameters used in a model fit to the observations. Moreover, since maximum-likelihood estimators ${ }^{3}$ enjoy the invariance property (see e.g., Mood et al. 1974, p. 284), $\varpi$ - and $\varpi^{\prime}$-fitting must yield identical results for those cases where $\varpi$-fitting yielded a positive parallax (since the $\varpi^{\prime}=\log \varpi$ transform may be used when $\varpi>0$ to extract $\varpi^{\prime}$ ).

Most of the objects considered in the present paper have large parallaxes that would have come out positive by a direct fit of $\varpi$ anyway. Thus, in the present case, the fitting of $\varpi^{\prime}$ (instead of $\varpi$ ) does not represent so much of an improvement. Nevertheless, the procedure of $\varpi^{\prime}$-fitting has been introduced here for the sake of generality.

The price to pay is, however, that the errors on $\varpi$ do not any longer follow a normal distribution. Moreover, the confidence interval of the parallax is no more symmetric. It has been estimated by the following expression:

$$
\begin{aligned}
& \hat{\varpi}^{\prime}-\sigma \leq \varpi^{\prime} \leq \hat{\varpi}^{\prime}+\sigma \\
& 10^{\hat{\varpi}^{\prime}-\sigma} \leq \varpi \leq 10^{\hat{\varpi}^{\prime}+\sigma}
\end{aligned}
$$

where $\hat{\varpi}^{\prime}$ is the value resulting from the $\chi^{2}$ - minimization, and $\sigma$ its estimated uncertainty. It should be noted that the errors on $\varpi^{\prime}$ do not follow a normal distribution either, since the normality is only guaranteed for "linear models" (Mood et al. 1974) and $\Xi$ (Eq. 5) does not depend linearly

\footnotetext{
${ }^{2}$ We are indebted to A. Albert for suggesting this trick.

${ }^{3} \chi^{2}$ is a maximum-likelihood estimator provided that the measurement errors are normally distributed.
} 
upon $\varpi^{\prime}$. Therefore, the confidence interval corresponding to a given probability level is in general not symmetric around $\varpi^{\prime}$. However, to ease the computations, the uncertainty $\sigma$ on $\varpi^{\prime}$ will be computed from the inverse of the Fisher information matrix at the point minimizing $\chi^{2}$ (see Sect. 2.1.3). This procedure implicitly assumes that the model is linear in the vicinity of the minimum, so that the adopted confidence interval is in fact one that is symmetric around $\varpi^{\prime}$.

The second expression in Eq. (9) clearly shows that the parallax is positive everywhere in the confidence interval, which would not be guaranteed in a constrained minimization of $\chi^{2}$. That important property illustrates the superiority of this approach with respect to the constrained minimization.

\subsection{3. $\chi^{2}$ minimization}

If the $\chi^{2}$ expressed by Eq. (5) were a quadratic expression of the unknown parameters $p_{k}$, its unique minimum could be found from the solution of a set of linear equations. However, the parameters $i, \omega$ and $\Omega$ enter $\chi^{2}$ in a highly non-linear way, so that the function expressing $\chi^{2}$ in the 9-parameter space may have several local minima, and finding its global minimum is a much more arduous task.

Faced with such situations, one of us (DP) has already successfully worked out global optimization techniques such as simulated annealing (Pourbaix 1994; Pourbaix 1998b). Practical details about the implementation of the method to minimize the objective function $\chi^{2}$ [Eq. (5)] in the working space $\mathbb{R}^{9}$ may be found in Pourbaix (1998a). Simulated annealing being a heuristic method, one can only prove its convergence to the global minimum after an infinite time (which we cannot afford). We thus stop the procedure after a finite time. In order to nevertheless have a good chance to obtain (a neighborhood of) the global minimum, we repeat 40 times this highly non deterministic minimization process. The best solution ever met (i.e., the one leading to the smallest $\chi^{2}$ value) is finally adopted. Once (a neighborhood of) the global minimum is thus obtained, it is tuned with the BFGS quasi-Newton algorithm (Dennis Jr. \& Schnabel 1995).

Unlike the Levenberg-Marquardt (Marquardt 1963) minimization algorithm, BFGS does not return the covariance matrix of the model parameters. The inverse of the Fisher information matrix at the minimum is therefore used as the best estimate of that covariance matrix (Pourbaix 1994).

The whole procedure has been applied separately on the data from the FAST consortium only, from NDAC only and from both combined, thus resulting in three different solutions, hopefully consistent with each other.

In a few instances, the solution obtained from the combined FAST+NDAC data set turns out to be very close to either the FAST or NDAC solution, but FAST and NDAC taken separately yield rather different solutions. That situation probably reflects the very different weights attributed to the two data sets for that particular object in the merging process applied to produce the output catalogue. For our analysis we always keep the covariance matrices of the observations as they are given in the electronic version of the catalogue.

As pointed out by an anonymous referee, in the case where all the Campbell elements $\left(a_{0}, i, \omega\right.$ and $\Omega$ ) are extracted from a fit to the astrometric data, they can advantageously be replaced by the Thiele-Innes elements $(A, B$, $F$ and $G$ ) so that $\chi^{2}$ becomes a quadratic function of the model parameters. The minimum of $\chi^{2}$ can then be found analytically and no minimization (neither global nor local) technique is needed. For the sake of generality, we nevertheless use the Campbell set (and thus the minimization scheme) because this more general scheme allows, if necessary, to easily incorporate external constraints (like for instance the knowledge of $i$ for eclipsing binaries, or $\omega$ from the spectroscopic orbit; see, however, the comment about fixing $\omega$ after Eq. (6) in Sect. 2.1.1). Such additional constraints would be much more difficult to impose through the Thiele-Innes elements.

\subsubsection{F-test: 5-parameter vs. orbital model}

The introduction of more free parameters in the orbital model as compared to the single star solution necessarily leads to a reduction of the objective function. To evaluate whether this reduction is statistically significant or, equivalently, whether the orbital solution represents a significant improvement over the 5-parameter Hipparcos solution - requires the use of an F-test.

The method used here is inspired from the test devised by Lucy \& Sweeney (1971). If $\chi_{\text {Hip }}^{2}$ and $\chi_{\text {orb }}^{2}$ denote the residuals for the Hipparcos 5-parameter model and for the orbital model (with 9 free parameters), respectively, the efficiency of the additional 4 parameters in reducing $\chi_{\text {orb }}^{2}$ below $\chi_{\text {Hip }}^{2}$ may be measured by the ratio:

$F=\frac{N-9}{4} \frac{\chi_{\text {Hip }}^{2}-\chi_{\text {orb }}^{2}}{\chi_{\text {orb }}^{2}}$,

where $N$ is the number of available measurements.

If the hypothesis that there is no orbital motion (i.e., $a_{0}=0$ in Eq. (5)) is correct, then it may be shown (Bevington \& Robinson 1992) that $F$ follows a Snedecor $F_{\nu_{1}, \nu_{2}}$ distribution with $\nu_{1}=4$ and $\nu_{2}=N-9$ degrees of freedom. Thus, if Eq. (10) yields $F=\hat{F}$, then, on the assumption that there is no orbital motion, the probability that $F$ could have exceeded $\hat{F}$ is

$\alpha=\operatorname{Prob}(F>\hat{F}) \equiv Q\left(\hat{F} \mid \nu_{1}, \nu_{2}\right)$.

In other words, $\alpha$ is the first risk error of rejecting the null hypothesis that the orbital and 5-parameter models are identical while it is actually true. 
The residuals given in the IAD files always relate to a 5-parameter solution, even when a more sophisticated model (the so-called "acceleration" 7- or 9-parameter models, or even orbital model) was published in the Hipparcos catalogue (see Table 1). For those cases, the $\alpha$ value listed in Table 1 is always close to zero, although it does not really characterize the improvement of the orbital solution with respect to the solution retained in the Hipparcos catalogue (which goes already beyond a 5parameter model).

\subsubsection{Astrophysical consistency of the orbital solution}

The minimization process will yield a solution in all cases, but that solution may not be astrophysically relevant. A statistical check of the significance of the orbital solution, based on the $F$-test, has been presented in Sect. 2.1.4. In this section, two criteria testing the validity of the orbital solution on astrophysical grounds are presented.

The first test is based upon the identity

$\frac{a_{0} \sin i}{\varpi}=\frac{P K_{1} \sqrt{1-e^{2}}}{2 \pi}$,

where $K_{1}$ is the radial-velocity semi-amplitude of the visible component. The left-hand side of the above identity entirely depends upon astrometric parameters, whereas its right-hand side contains only parameters derived spectroscopically.

This test has the advantage of being totally independent of any assumptions. However, it involves the orbital inclination which is not always very accurately determined (see Sect. 3.2), so that the above identity may not always be very constraining considering the often large uncertainty on $i$.

A somewhat more constraining identity to assess the astrophysical plausibility of the computed astrometric orbit is the following:

$\frac{a_{0}}{\varpi}=P^{2 / 3} \frac{M_{2}}{\left(M_{1}+M_{2}\right)^{2 / 3}}$,

where $M_{1}$ and $M_{2}$ are the masses of the visible and invisible components, respectively. However, it relies upon assumptions regarding the stellar masses. In some cases (e.g., dwarf barium stars), the mass of the observable component may be estimated directly from the spectroscopically-derived gravity and from an estimate of the stellar radius from the spectral type (North et al. 1999). For the other samples, an average mass derived from a statistical analysis of the spectroscopic mass function (Jorissen et al. 1998) is adopted. For all the samples considered here, the unseen component is almost certainly a white dwarf (the only possible exception being the Tc-poor S star HIP $99312=$ HD 191589), whose mass may be taken as $0.62 \pm 0.04 M_{\odot}$ (Jorissen et al. 1998).

Because of the assumptions involved, this test is only used as a guide to identify astrophysically-unplausible solutions. It turns out that such cases are generally those with large error bars or with inconsistent N, F and A solutions, thus providing further arguments not to retain those solutions. In very few cases (HIP 36042, 53763 and 60299), valid data yielded solutions not consistent with Eq. (13). Those cases were nevertheless kept in our final list.

\subsection{Transit Data}

Unlike the IAD, TD are only available for a small subset of the Hipparcos catalogue, e.g., for those stars that were known to be (or suspected of being) double or multiple systems at the time of the data reduction by the Hipparcos consortia. TD are a by-product of (or, more precisely, an input for) the multiple-star processing by the NDAC consortium. Another difference with respect to the IAD concerns photometry. Whereas IAD contain astrometric information only, the brightness of the (different) observable component(s) of the system can be retrieved from the TD.

In the most general case (Quist \& Lindegren 1999), each entry in the TD file corresponds to five numbers $b_{1}, \ldots, b_{5}$ which represent the coefficients of the first terms in the Fourier series modeling the observed signal as modulated by the detector grid:

$$
\begin{aligned}
& b_{1}=\sum_{i} I_{i} \\
& b_{2}=\tilde{M} \sum_{i} I_{i} \cos \phi_{i} \\
& b_{3}=-\tilde{M} \sum_{i} I_{i} \sin \phi_{i} \\
& b_{4}=\tilde{N} \sum_{i} I_{i} \cos \left(2 \phi_{i}\right) \\
& b_{5}=-\tilde{N} \sum_{i} I_{i} \sin \left(2 \phi_{i}\right)
\end{aligned}
$$

where $I_{i}$ is the intensity of the $i$-th component of the system, and the phase $\phi_{i}$ corresponds to its abscissa along the reference great circle. $\tilde{M}=0.7100$ and $\tilde{N}=0.2485$ are the adopted reference values for the modulation coefficients of the first and second harmonics (Vol. 1, Sect. 2.9; ESA 1997). In terms of the Cartesian coordinates $\left(\xi_{i}, \eta_{i}\right)$ (see Eq. (3)) of component $i$ in the plane tangent to the sky at the reference point specified in the TD file, the phase $\phi_{i}$ writes

$\phi_{i}=f_{x} \xi_{i}+f_{y} \eta_{i}+f_{\mathrm{p}}\left(\varpi-\varpi_{\mathrm{ref}}\right)$.

The reference point is assigned an arbitrary parallax $\varpi_{\text {ref }}$ and proper motion, as given in the TD file. The phase derivatives $f_{x}, f_{y}$ and $f_{\mathrm{p}}$ with respect to $\xi, \eta$ and $\varpi$ are also provided by the TD file.

For the SB1 systems we are interested in, the situation simplifies a lot since $I_{2}$ may be taken equal to 0 . The above 
Table 1. The various samples of CPRS that have been considered in the present work, along with various parameters characterizing the quality of the orbital solution derived from the IAD (see text for details). $\varpi_{\text {HIP }}$ is the parallax from the Hipparcos catalogue, $N_{\text {IAD }}$ is the number of IAD available for the star considered, $\beta$ is the ecliptic latitude, $T$ is the time of periastron

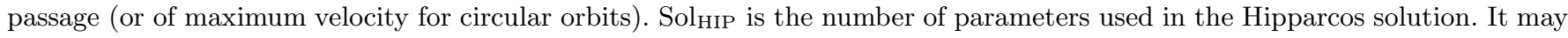
be one of the following types: " $5 "=5$-parameter solution, "7" = 7-parameter solution, i.e., including a linear acceleration term in the proper motion (see Sect. 2.3, Vol. 1 of ESA 1997), "9" = 9-parameter solution, i.e., including a quadratic acceleration term in the proper motion ("7"- and "9"-entries are listed in the "G" section of the Double and Multiple Star Annex - DMSA of the Hipparcos and Tycho Catalogues), "X" = stochastic solution (described in DMSA/X), and "O" = orbital solution given in the DMSA/O. The column labeled "Transit?" indicates whether TD are also available for the corresponding star. The column labeled $\chi^{2} /\left(N_{\text {IAD }}-9\right)$ lists the unit-weight variance for the orbital-parameter model. $\alpha$ is the first risk error of rejecting the null hypothesis that the orbital and single star models are identical while it is actually true (Eq. 11). The expected value of the orbital separation $a_{0} / \varpi$ is computed from Eq. (13). The $1 \sigma$ confidence interval is given next to the computed astrometric $a_{0} / \varpi$ value. Column "Rem" indicates whether the orbital solution derived from the IAD has been accepted. An asterisk in column "Rem" refers to a note at the bottom of the table

\begin{tabular}{|c|c|c|c|c|c|c|c|c|c|c|c|c|c|c|}
\hline HIP & $\mathrm{HD} / \mathrm{DM}$ & $\begin{array}{l}\varpi_{\text {HIP }} \\
\text { (mas) }\end{array}$ & $N_{\text {IAD }}$ & \multicolumn{2}{|c|}{$\begin{array}{ll}\beta & P \\
\left.{ }^{\circ}\right) & (\mathrm{d}) \\
\end{array}$} & $e^{e} \quad(\mathrm{~J}$ & \multicolumn{2}{|c|}{$\begin{array}{cc}T & \text { Sol }_{\text {HIP }} \\
\text { JD- } 2 & 2000000) \\
\end{array}$} & Transit? & $\frac{\chi^{2}}{\left(N_{\text {IAD }}-9\right)}$ & $\begin{array}{l}\alpha \\
(\%) \\
\end{array}$ & \multicolumn{2}{|c|}{$a_{0} / \varpi$} & Rem \\
\hline \multicolumn{15}{|c|}{ CH stars } \\
\hline 168 & 224959 & $1.95 \pm 1.34$ & 62 & -2.8 & 1273.0 & 0.179 & 46064 & 5 & $\mathrm{y}$ & 1.23 & 36 & $1.6_{0.41}^{5.1}$ & $1.08 \pm 0.53$ & \\
\hline 4252 & 5223 & $1.12 \pm 1.17$ & 50 & 16.8 & 755.2 & 0 & 45535.6 & 5 & & 0.69 & 0 & $1 \mathrm{e}+09 \mathrm{0}^{+\infty}$ & $0.76 \pm 0.38$ & \\
\hline 22403 & 30443 & $1.63 \pm 1.7$ & 28 & 12.5 & 2954.0 & 0 & 46306 & 5 & $\mathrm{y}$ & 0.90 & 9 & $24 \begin{array}{l}67 \\
3.2\end{array}$ & $1.89 \pm 0.94$ & \\
\hline 53763 & $+42^{\circ} 2173$ & $5.29 \pm 1.47$ & 70 & 32.2 & 328.3 & 0 & 46542.1 & 5 & & 0.84 & 3 & $1.8 \begin{array}{l}8.3 \\
0.3\end{array}$ & $0.44 \pm 0.22$ & Accepted, ${ }^{*}$ \\
\hline 62827 & $+8^{\circ} 2654 \mathrm{~A}$ & $5.3 \pm 2.15$ & 40 & 11.8 & 571.1 & 0 & 46467.8 & 5 & & 1.10 & 89 & $0.42{ }_{0}^{1.5}$ & $0.63 \pm 0.31$ & $*$ \\
\hline 102706 & 198269 & $3.16 \pm 1.11$ & 68 & 34.2 & 1295.0 & 0.094 & 46358 & 5 & & 1.18 & 34 & $2.5 \begin{array}{l}5.6 \\
0.78\end{array}$ & $1.09 \pm 0.54$ & \\
\hline 104486 & 201626 & $4.93 \pm 0.84$ & 100 & 40.6 & 1465.0 & 0.103 & 45970 & 7 & & 1.35 & 0 & $1.5 \begin{array}{l}2.3 \\
0.84\end{array}$ & $1.18 \pm 0.58$ & Accepted \\
\hline 108953 & 209621 & $1.47 \pm 1.3$ & 46 & 30.6 & 407.4 & 0 & 45858.3 & 5 & & 1.02 & 94 & $0.8 \begin{array}{l}9.3 \\
0\end{array}$ & $0.50 \pm 0.25$ & \\
\hline \multicolumn{15}{|c|}{ Dwarf Ba stars } \\
\hline 8647 & 11377 & $6.38 \pm 1.14$ & 42 & -26.3 & 4140.0 & 0.16 & 45240 & 5 & $\mathrm{y}$ & 0.95 & 34 & $9.5_{0}^{27}$ & $2.20 \pm 0.97$ & \\
\hline 32894 & 50264 & $14.11 \pm 1.96$ & 78 & -52.2 & 912.4 & 0.098 & 46791 & $\mathrm{X}$ & & 1.05 & 0 & $0.84{ }_{0.7}^{1}$ & $0.89 \pm 0.46$ & Accepted \\
\hline 49166 & 87080 & $7.9 \pm 1.39$ & 90 & -42.2 & 273.4 & 0.177 & 48373 & 5 & & 1.03 & 34 & $0.47 \begin{array}{l}0.85 \\
0.2\end{array}$ & $0.39 \pm 0.19$ & Accepted \\
\hline 50805 & 89948 & $23.42 \pm 0.93$ & 60 & -36.6 & 667.8 & 0.117 & 46918 & $\mathrm{O}$ & $\mathrm{y}$ & 0.82 & 0 & $0.67 \begin{array}{l}0.73 \\
0.6\end{array}$ & $0.67 \pm 0.30$ & Accepted \\
\hline 60299 & 107574 & $5.02 \pm 1.06$ & 50 & -14.7 & 1350.0 & 0.081 & 46342 & 9 & & 0.93 & 0 & $1.8_{1.2}^{2.5}$ & $0.83 \pm 0.26$ & Accepted \\
\hline 62409 & $+17^{\circ} 2537$ & $8.2 \pm 1.28$ & 58 & 20.1 & 1796.0 & 0.14 & 46291 & 5 & & 1.29 & 25 & $0.87 \begin{array}{l}1.7 \\
0.28\end{array}$ & $1.34 \pm 0.65$ & NDAC accepted \\
\hline 69176 & 123585 & $8.75 \pm 1.39$ & 54 & -29.3 & 457.8 & 0.062 & 48207 & 5 & & 1.24 & 0 & $0.47 \begin{array}{c}0.69 \\
0.31\end{array}$ & $0.44 \pm 0.16$ & Accepted \\
\hline 71058 & 127392 & $10.63 \pm 1.7$ & 46 & -15.4 & 1498.7 & 0.071 & 47070 & 5 & & 0.85 & 73 & $0.56{ }_{0}^{1.4}$ & $1.21 \pm 0.61$ & \\
\hline 104785 & 202020 & $9.53 \pm 1.5$ & 52 & 6.1 & 2064.0 & 0.08 & 47122 & 5 & & 1.16 & 14 & $1.4 \begin{array}{l}3.2 \\
0.14\end{array}$ & $1.51 \pm 0.77$ & \\
\hline 105969 & 204613 & $16.61 \pm 1.78$ & 80 & 64.8 & 878.0 & 0.13 & 47479 & $\mathrm{X}$ & & 1.02 & 0 & $0.75 \begin{array}{c}0.84 \\
0.66\end{array}$ & $0.77 \pm 0.33$ & Accepted \\
\hline 107818 & 207585 & $7.53 \pm 1.51$ & 52 & -10.5 & 670.6 & 0.03 & 47319 & 5 & & 0.82 & 7 & $0.39 \begin{array}{l}0.65 \\
0.2\end{array}$ & $0.58 \pm 0.22$ & Accepted \\
\hline 116233 & 221531 & $8.83 \pm 1.21$ & 56 & -8.3 & 1416.0 & 0.165 & 47157 & 7 & & 0.84 & 0 & $\begin{array}{ll}1.2 & 1.5 \\
0.89\end{array}$ & $0.97 \pm 0.37$ & Accepted \\
\hline 118266 & 224621 & $6.95 \pm 1.45$ & 80 & -32.6 & 307.8 & 0.048 & 49345 & 5 & & 1.00 & 45 & $\begin{array}{ll}0.27 & 0.55 \\
0.081\end{array}$ & $0.40 \pm 0.19$ & \\
\hline
\end{tabular}

Remarks:

HIP 53763 \& 62827: large uncertainty on $\varpi$ in the 9-parameter solution (see Table 2), since the orbital period is close to 1 yr.

system of equations is rank-deficient. From the second and and also express the uncertainty on $\phi$ as third equations, one can rewrite:

$\stackrel{\mathrm{o}}{\phi}=\arg \left(b_{2},-b_{3}\right)$

$$
\sigma_{\phi}^{2}=\left(\frac{\partial \stackrel{\circ}{\phi}}{\partial b_{2}}\right)^{2} \sigma_{b_{2}}^{2}+\left(\frac{\partial \stackrel{\circ}{\phi}}{\partial b_{3}}\right)^{2} \sigma_{b_{3}}^{2}
$$


Table 1. continued

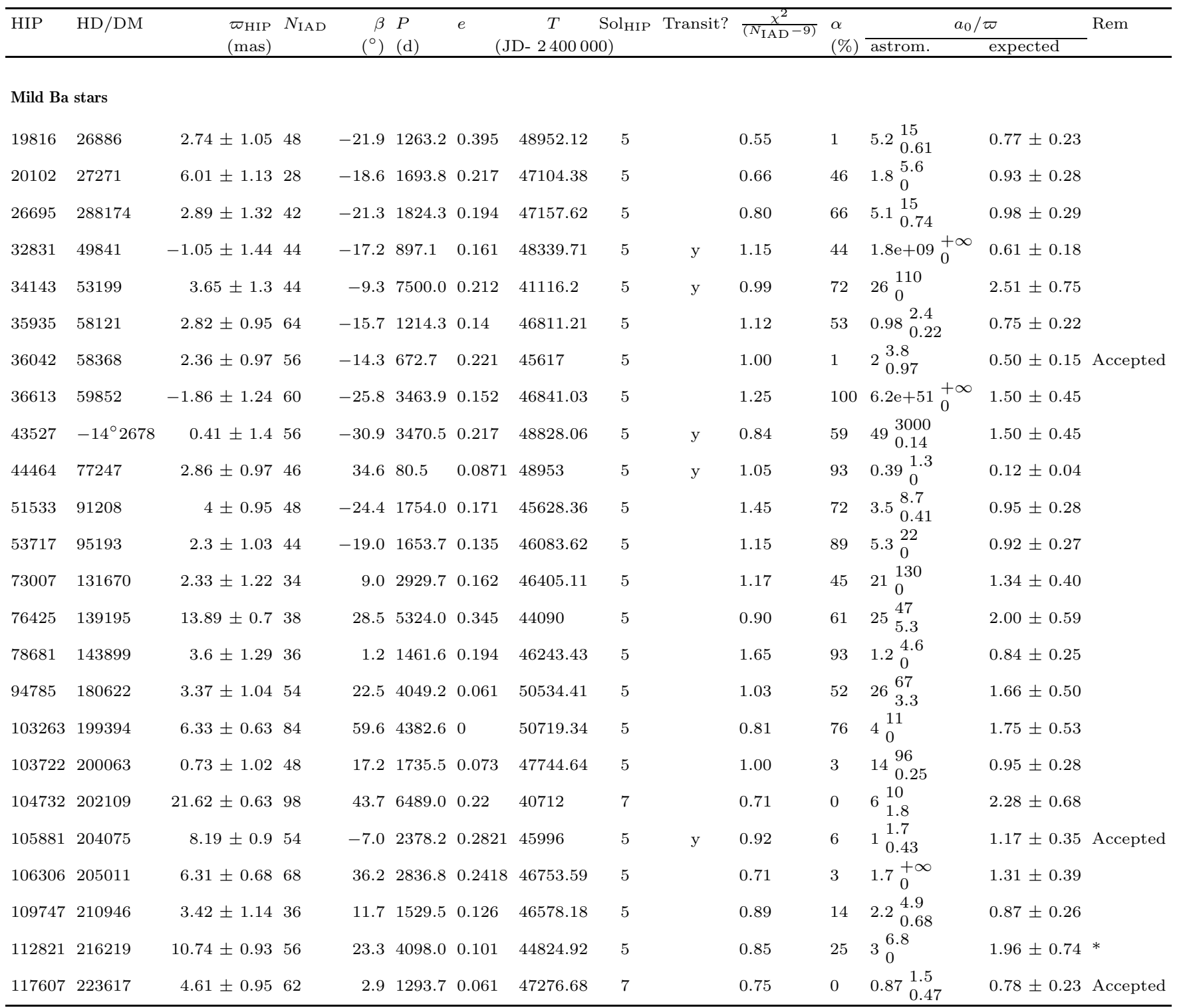

Rem: HIP 112821 is also sometimes classified as a dwarf barium star.

With the above definitions, the objective function whose minimum is sought is given by:

$D=\frac{1}{N} \sum_{k=1}^{N} \sigma_{\phi_{k}}^{-2}\left(\stackrel{\circ}{\phi_{k}}-\phi_{k}\right)^{2}$

where $N$ is the number of TD. In the above expression, $\stackrel{\circ}{\phi_{k}}$ is the observed phase at time $t_{k}$ as derived from Eq. (15), whereas $\phi_{k}$ is the phase computed from Eqs. (3) and (14) (noting that $f_{x} R P_{\alpha}+f_{y} R P_{\delta} \equiv f_{\mathrm{p}}$ ) for a given set of astrometric and orbital parameters.

The remaining of the method follows the same steps as described in relation with the IAD, i.e. global and local optimization, positiveness of the parallax, ...

\section{Results}

Table 1 lists the various samples that have been considered, namely dwarf barium and subgiant $\mathrm{CH}$ stars (McClure 1997; North, priv. comm.), mild and strong barium stars (Jorissen et al. 1998), Tc-poor S stars (Jorissen et al. 1998) and CH stars (McClure \& Woodsworth 1990). Their spectroscopic orbital parameters have been taken from the reference quoted.

Table 1 also provides various parameters that allow either to assess the quality of the derived orbital solution or to identify the reason why a reliable orbital solution could not be derived for some of the spectroscopic binaries considered. The following parameters potentially control our ability to derive an astrometric orbit from the IAD, with favorable circumstances being mentioned between 
Table 1. continued

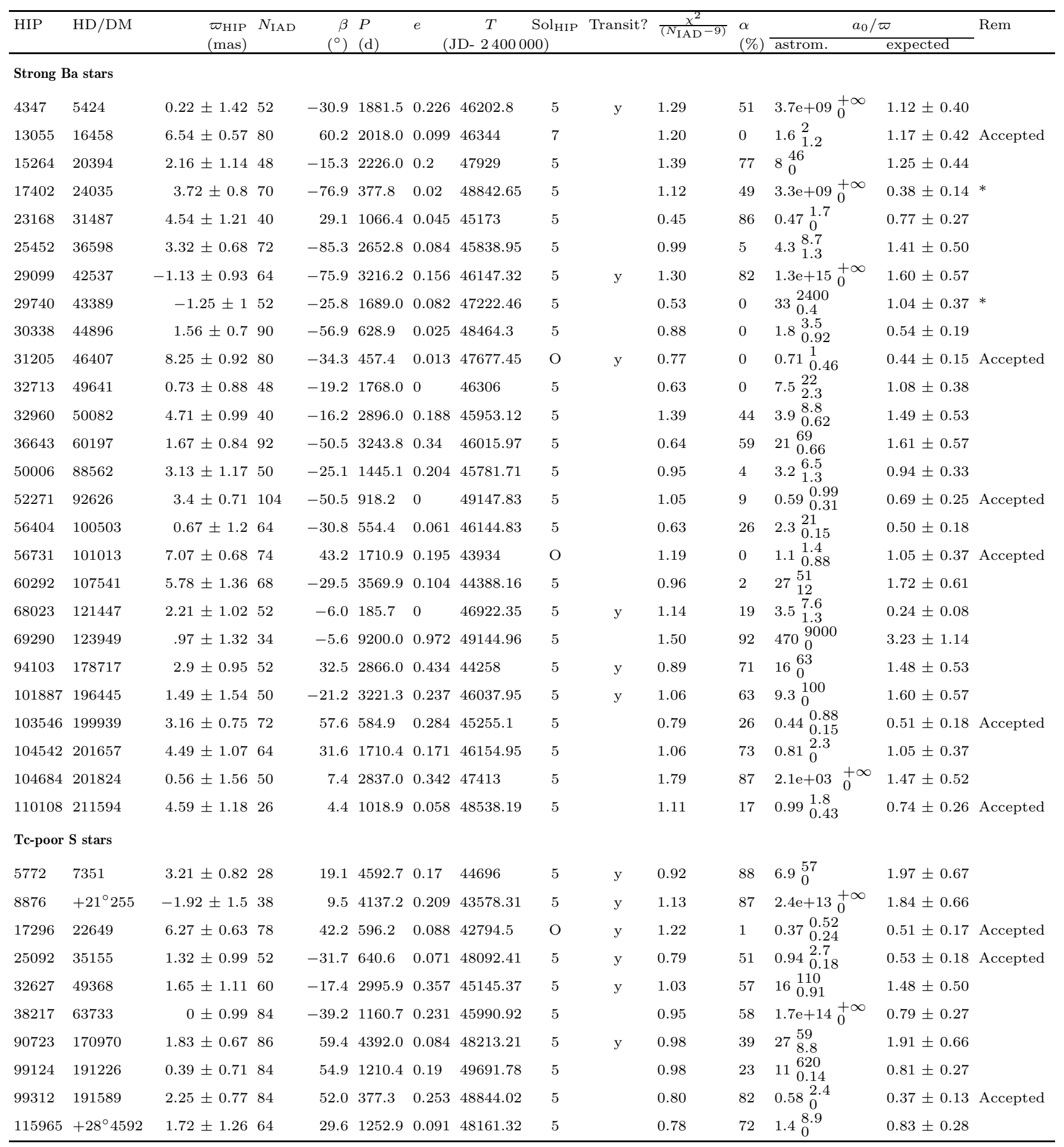

HIP 29740: $\alpha=0$ but the solutions are totally unrealistic.

HIP 17402: $\varpi$ not accurately determined, since orbital period is close to $1 \mathrm{yr}$. 
the parentheses: the parallax (Col. 3; large parallax), the number of available IAD measurements (FAST+NDAC; Col. 4 ; large number of measurements), the ecliptic latitude (Col. 5; this parameter may play a role since it controls how different the orientations of the reference great circles are; favorable cases have absolute values larger than $47^{\circ}$ ), the orbital period (Col. 6 ; in the range $1-3$ yr to ensure a good sampling of the orbit), the orbital eccentricity (Col. 7; low eccentricity).

The following columns characterize the quality of the solution obtained from the minimization process. Column 11 provides $\chi^{2} /\left(N_{\text {IAD }}-9\right)$, which should be of the order of unity if the internal error $\sigma$ (Eq. 2) on the abscissae has been correctly evaluated by the reduction consortia and if the model provides an adequate representation of the data. The first risk error associated with rejecting the null hypothesis that the orbital and Hipparcos 5-parameter solutions are identical (Eq. 11) is given in Col. 12. Low values of $\alpha$ are generally associated with Hipparcos solutions of the $\mathrm{G}, \mathrm{X}$ or $\mathrm{O}$ types (as listed in Col. 9; see the caption to Table 1 for more details), since the orbital motion is then large enough to have been noticed already by NDAC or FAST. Columns 13 and 14 compare the $a_{0} / \varpi$ ratio derived from the orbital solution to its expected value from Eq. (13). In Cols. 11 to 13, the data refer to the orbital solution obtained by combining NDAC and FAST data. For dwarf barium stars, the masses used to estimate $a_{0} / \varpi$ according to Eq. (13) are $M_{2}=0.62 \pm 0.04 M_{\odot}$, whereas $M_{1}$ is derived from the spectroscopic gravity, with an estimated error of $0.05 M_{\odot}$ (North, priv. comm.). According to the statistical analysis of the spectroscopic mass functions performed by Jorissen et al. (1998), $M_{1}$ and $M_{2}$ pairs (expressed in $\left.M_{\odot}\right)$ of $(1.7 \pm 0.2,0.62 \pm 0.04)$, $(2.1 \pm 0.2,0.62 \pm 0.04)$ and $(1.8 \pm 0.2,0.62 \pm 0.04)$ have been adopted for strong barium stars, mild barium stars and Tc-poor S stars, respectively. The same analysis performed by McClure \& Woodsworth (1990) for CH stars yielded $M_{1}=1.0 \pm 0.1 M_{\odot}$ and $M_{2}=0.62 \pm 0.04 M_{\odot}$.

Astrometric orbits were accepted when $\alpha$ is smaller than 10 per cent and the expected $a_{0} / \varpi$ value falls within the $1 \sigma$ confidence interval. A few cases not fulfilling these criteria were nevertheless accepted after visual inspection of the orbital arc.

Examination of Table 1 reveals that the following criteria need to be fulfilled in order to be able to extract a reliable astrometric orbit from the Hipparcos data: $\varpi \gtrsim 5$ mas, $P \lesssim 10 \mathrm{yr}, N \gtrsim 25$. The success rate is as follows for the various samples: dwarf barium stars $(9 / 13)$, mild barium stars $(3 / 24)$, strong barium stars $(6 / 26)$, Tcpoor S stars $(3 / 10)$ and $\mathrm{CH}$ stars $(2 / 8)$. The high success rate for dwarf barium stars naturally results from the fact that these dwarf stars are on average closer from the sun than the giant stars.

Table 2 lists the astrometric and orbital parameters for the reliable orbits according to the criteria discussed above. The results from the different processing modes are collected in Table 2, according to the symbol given in Col. 2: H/S refers to the parameters from the Hipparcos catalogue and from the spectroscopic orbit [on that line $a_{0} / \varpi$ is the semi-major axis in A.U. estimated from Eq. (13)], F refers to the processing of the IAD from FAST only, $\mathrm{N}$ to the IAD from NDAC only, A from the processing of the combined FAST/NDAC data set, $\mathrm{O}$ to the orbital parameters from the DMSA/O, and T to the parameters resulting from the TD.

Most of the retained orbits are indeed characterized by $\chi^{2} /(N-9)$ values of the order of unity, as expected. The first-risk errors $\alpha$ are not always close to 0 , but if the derived value for the semi-major axis is in good agreement with its expected value, that agreement has been considered as sufficient for retaining the orbit. The only cases where the reverse situation occurs (small $\alpha$ but discrepant $\left.a_{0} / \varpi\right)$ are the dwarf barium star HIP 60299, the mild barium star HIP 36042 and the CH star HIP 53763. Although the orbit of the latter is not well defined, it has been kept in our final list to illustrate the large uncertainty on $\varpi$ resulting from an orbital period close to $1 \mathrm{yr}$ (Sect. 4).

The F, N and A solutions for the retained orbits are also generally in good agreement, the only exceptions being the dwarf Ba stars HIP 62409 and HIP 116233, and the mild Ba star HIP 117607. However, the model parameters of these systems are highly correlated, and the different measurement errors in the different data sets thus drive the solution in different directions. This statement may be expressed in a quantitative way using the concept of efficiency $\epsilon$ introduced by Eichhorn (1989) and Pourbaix \& Eichhorn (1999). It is defined as

$\epsilon=\sqrt[p]{\frac{\prod_{k=1}^{p} \lambda_{k}}{\prod_{k=1}^{p} q_{k k}}}$

where $\lambda_{k}$ are the eigenvalues of the covariance matrix of the estimated parameters, $q_{k k}$ are its diagonal elements, and $p$ denotes the number of parameters in the model. If $\epsilon$ is close to unity, there is obviously little correlation between the parameters. For the combined NDAC+FAST solution, it amounts to $0.32,0.21$ and 0.42 in the case of HIP 62409, HIP 116233 and HIP 117607, respectively, thus translating some degree of correlation between the model parameters.

In Table 2 , the uncertainty on $a_{0} / \varpi$ has been computed by combining the upper and lower limits on $a_{0}$ and $\varpi$, thus neglecting any possible correlation between these two quantities (which is generally small - except for the three systems listed above - as derived from the efficiency being close to unity).

The orbital solutions derived in the present paper are too many to display the astrometric orbit for all cases. A few representative cases among the different subsets of Table 1 (orbital periods shorter or longer than the duration of the Hipparcos mission, small or large 
Table 2. Astrometric orbital elements for various classes of CPRS. The first line provides the data from the HIPPARCOS catalogue or from the spectroscopic orbit (label H/S in Col. 2). The following lines provide the orbital solutions derived in the present work from the FAST, NDAC and combined FAST+NDAC IAD (labels F, N and A, respectively in Col. 2). When available, the orbital solution from the transit data (label T in Col. 2) or from the DMSA/O (label O) are given next. The columns $\varpi, a_{0} / \varpi$ and $K_{1}$ contain the nominal value followed by the boundaries of the " $1 \sigma$ " confidence interval (computed by propagating the $1 \sigma$ errors on $a_{0}, \varpi^{\prime}$ and $i$ as if they were uncorrelated). An asterisk after the HIP number refers to a note at the end of the table

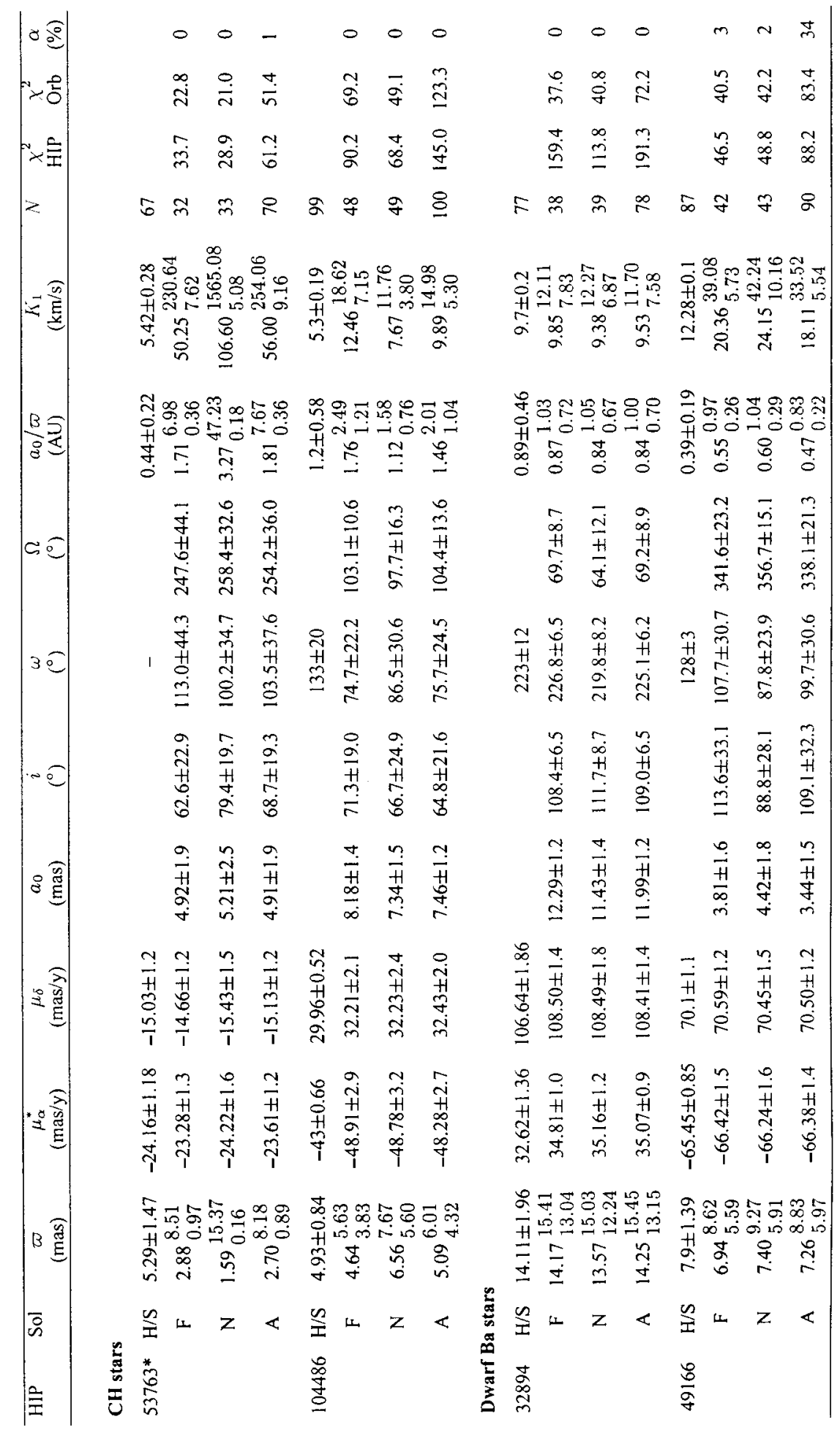


Table 2. continued

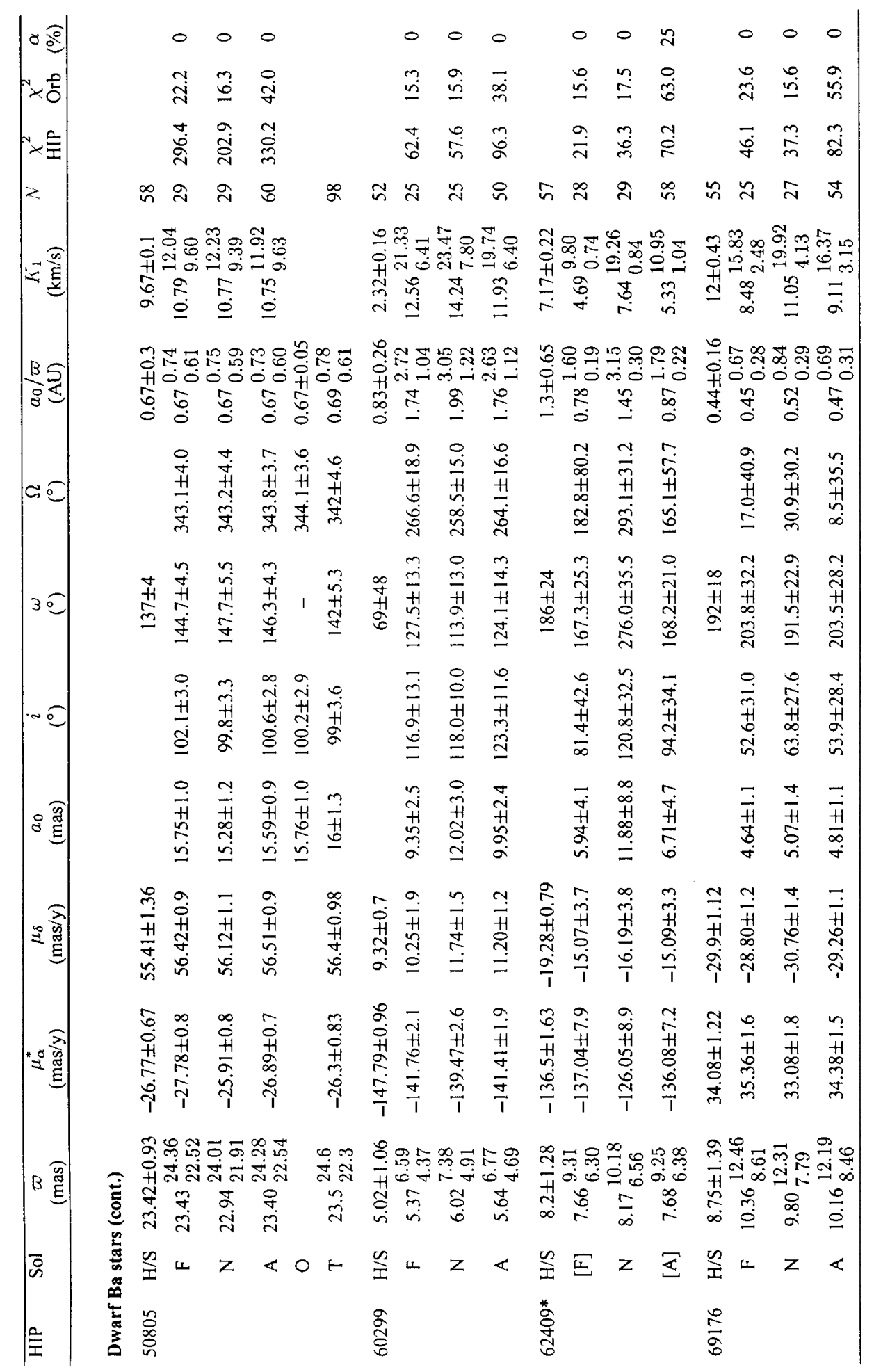


Table 2. continued

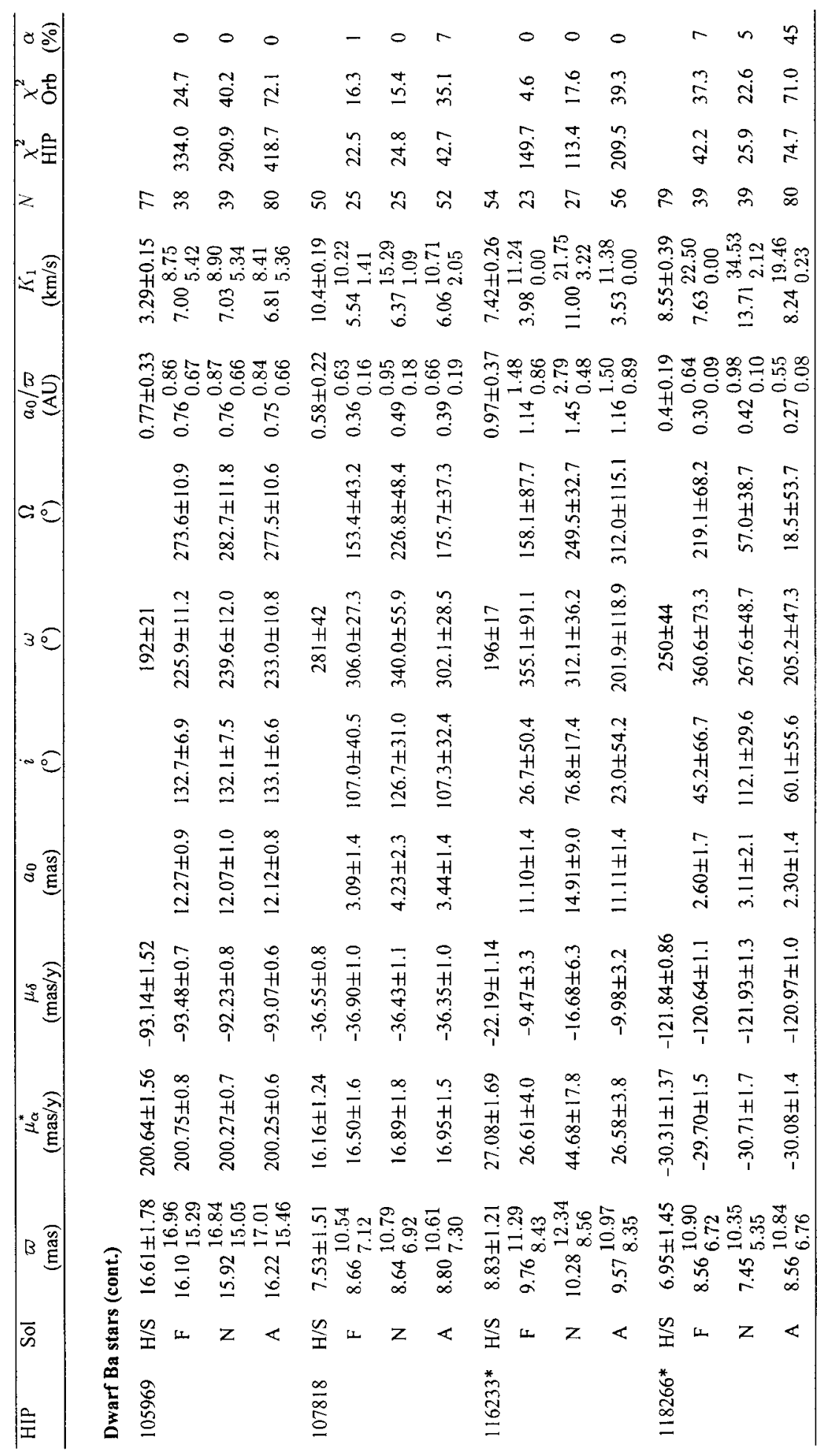


Table 2. continued

\begin{tabular}{|c|c|c|c|c|c|c|c|c|c|c|c|c|c|c|}
\hline 0 (2) & & & 0 & 0 & - & & 0 & 0 & 0 & & & 0 & 0 & 0 \\
\hline$\times 50$ & & & $\begin{array}{l}\infty \\
\stackrel{\infty}{i}\end{array}$ & $\mathfrak{\infty}$ & aे & & $\begin{array}{l}\mathscr{\infty} \\
\propto \\
-\end{array}$ & $\stackrel{\infty}{\simeq}$ & $\stackrel{\forall}{\dot{J}}$ & & & $\stackrel{n}{\infty}$ & $\begin{array}{l}\stackrel{0}{i} \\
\text { in }\end{array}$ & $\stackrel{a}{g}$ \\
\hline$\times \hat{\Xi}$ & & & $\vec{m}$ & $\stackrel{\circ}{\infty}$ & $\vec{b}$ & & $\begin{array}{l}\infty \\
\stackrel{8}{े}\end{array}$ & 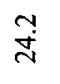 & $\tilde{B}$ & & & $\dot{\Xi}$ & $\stackrel{n}{\equiv}$ & ڤ̊ \\
\hline$z$ & & in & $\stackrel{\infty}{\sim}$ & 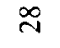 & $i$ & $\bar{n}$ & $\approx$ & $\stackrel{\sim}{\sim}$ & $\dot{n}$ & $\infty$ & 8 & શิ & $\bar{n}$ & $\widetilde{\delta}$ \\
\hline$=\frac{\widehat{D}}{\mathrm{a}}$ & & & 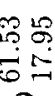 & \begin{tabular}{l}
$\infty$ \\
\hdashline \\
\hdashline \\
\hdashline
\end{tabular} & 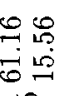 & & 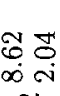 & 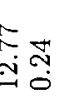 & نר & & & & 学 & \\
\hline & & $\frac{a}{\sigma}$ & 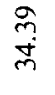 & $\begin{array}{l}\infty \\
\infty \\
\ddot{j}\end{array}$ & 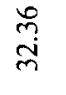 & 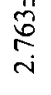 & $\begin{array}{l}\tilde{O} \\
\stackrel{n}{n}\end{array}$ & $\begin{array}{l}\stackrel{\circ}{\circ} \\
\dot{\sigma}\end{array}$ & $\underset{\forall}{\nabla}$ & & $\stackrel{6}{6}$ & $\frac{\infty}{6}$ & $\begin{array}{l}\bar{\sigma} \\
\dot{ \pm}\end{array}$ & $\stackrel{\cong}{N}$ \\
\hline & & & $F=$ & 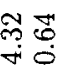 & 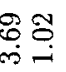 & & & 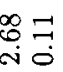 & $\begin{array}{l}8 \pi \\
\end{array}$ & PO & & مَ & 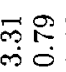 & 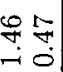 \\
\hline & & $\stackrel{H}{2}$ & $\underset{\mathrm{i}}{\stackrel{\infty}{\circ}}$ & $\stackrel{\infty}{\stackrel{0}{=}}$ & g & $\stackrel{+}{\stackrel{H}{\longrightarrow}}$ & $\stackrel{\infty}{\circ}$ & 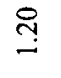 & $\stackrel{8}{8}$ & $\stackrel{0}{-}$ & $\stackrel{\substack{1 \\
0}}{0}$ & $\stackrel{\substack{0 \\
0}}{0}$ & $\stackrel{ \pm}{\stackrel{\Xi}{Z}}$ & 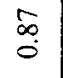 \\
\hline & & & $\begin{array}{l}\bar{\infty} \\
+1 \\
\bar{\infty} \\
\stackrel{\sim}{d}\end{array}$ & 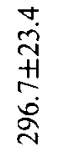 & $\begin{array}{l}\stackrel{\sigma}{\alpha} \\
+1 \\
\infty \\
\stackrel{\alpha}{\alpha}\end{array}$ & & $\begin{array}{l}\stackrel{+}{n} \\
\stackrel{+}{+} \\
+0 \\
\stackrel{\infty}{\infty}\end{array}$ & 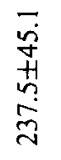 & $\begin{array}{l}\stackrel{\circ}{\circ} \\
\stackrel{5}{H} \\
+ \\
\stackrel{2}{0}\end{array}$ & 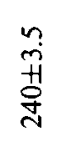 & & $\begin{array}{l}n \\
\stackrel{n}{n} \\
+1 \\
r \\
\infty \\
\stackrel{0}{n}\end{array}$ & $\begin{array}{l}0 \\
\infty \\
\infty \\
+ \\
\infty \\
\dot{J} \\
\dot{v}\end{array}$ & 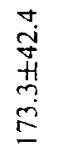 \\
\hline & & 0 & $\dot{m}$ & $\ddot{0}$ & $\stackrel{g}{ \pm}$ & $\frac{O}{H}$ & $\tilde{n}$ & $\stackrel{\sim}{\sim}$ & $\stackrel{\infty}{\stackrel{\infty}{\varrho}}$ & $\overrightarrow{r i}$ & $\stackrel{\mathscr{I}}{+}$ & $\begin{array}{l}\dot{0} \\
\ddot{n}\end{array}$ & $\overline{\bar{\lambda}}$ & $\begin{array}{r}\dot{v} \\
\ddot{v}\end{array}$ \\
\hline & & $\stackrel{H}{I}$ & $\frac{+}{\infty}$ & $\frac{+}{\forall}$ & 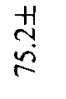 & $\frac{\bar{n}}{\stackrel{n}{n}}$ & 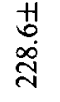 & $\begin{array}{l}+ \\
\stackrel{+}{0} \\
\stackrel{d}{\circ}\end{array}$ & $\begin{array}{l}+ \\
\infty \\
\stackrel{2}{*} \\
\stackrel{\sim}{*}\end{array}$ & $\frac{H}{D}$ & $\begin{array}{l}\frac{11}{6} \\
5 \\
n\end{array}$ & $\frac{H}{a}$ & 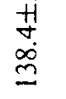 & $\begin{array}{l}+1 \\
0 \\
\dot{+} \\
\end{array}$ \\
\hline & & & $\begin{array}{l}\stackrel{2}{0} \\
+0 \\
\dot{0}\end{array}$ & $\begin{array}{l}\hat{N} \\
\tilde{N} \\
+1 \\
\stackrel{a}{=}\end{array}$ & $\begin{array}{l}n \\
\bar{n} \\
+ \\
\frac{1}{0}\end{array}$ & & 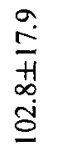 & 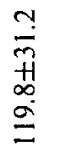 & 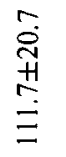 & $\begin{array}{l}\stackrel{0}{\mathrm{i}} \\
\stackrel{H}{H} \\
\stackrel{\Xi}{\Xi}\end{array}$ & & $\begin{array}{l}\infty \\
\infty \\
\stackrel{0}{0} \\
\dot{H} \\
\stackrel{0}{0} \\
=\end{array}$ & $\begin{array}{l}n \\
\tilde{n} \\
+H \\
\infty \\
\infty \\
\infty\end{array}$ & $\begin{array}{l}\vec{r} \\
\tilde{n} \\
+ \\
m \\
\dot{n}\end{array}$ \\
\hline $8 \stackrel{g}{g}$ & & & $\begin{array}{l}\frac{1}{H} \\
\frac{H}{\sigma} \\
\text { i }\end{array}$ & $\underset{m}{\frac{m}{n}}$ & $\begin{array}{l}= \\
\text { Ḣ } \\
\infty \\
\infty \\
+ \\
+\end{array}$ & & $\begin{array}{l}\stackrel{r}{+} \\
\stackrel{+}{E} \\
\stackrel{\sigma}{\sigma}\end{array}$ & $\begin{array}{l}\stackrel{\eta}{\infty} \\
H \\
\stackrel{\sim}{\sim} \\
\sigma\end{array}$ & 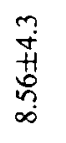 & $\begin{array}{l}\stackrel{2}{\infty} \\
0 \\
\stackrel{+}{H} \\
\stackrel{2}{0}\end{array}$ & & $\begin{array}{l}\text { ב } \\
\text { H } \\
\sim \\
n \\
n\end{array}$ & $\begin{array}{l}0 \\
\sim \\
+ \\
\infty \\
\infty \\
0 \\
0\end{array}$ & $\frac{0}{H}$ \\
\hline 焉 & & \begin{tabular}{l}
$\overline{0}$ \\
$\stackrel{0}{+}$ \\
+ \\
\multirow{H}{*}{} \\
$\dot{0}$
\end{tabular} & 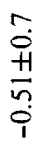 & $\begin{array}{l}\hat{0} \\
\dot{0} \\
\dot{0} \\
\hat{0} \\
0 \\
1\end{array}$ & $\frac{\stackrel{0}{0}}{\stackrel{+}{H}}$ & $\begin{array}{l}\underset{+}{0} \\
\stackrel{+}{+} \\
\infty \\
\infty \\
\infty \\
\infty\end{array}$ & 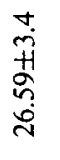 & $\begin{array}{l}\infty \\
\underset{n}{+} \\
\stackrel{\sim}{n}\end{array}$ & $\begin{array}{l}\bar{n} \\
+\overrightarrow{0} \\
\text { in } \\
\text { in }\end{array}$ & 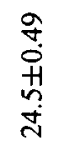 & 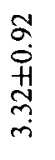 & $\frac{+}{\frac{+}{n}}$ & $\begin{array}{l}\stackrel{0}{-} \\
\text { H } \\
\\
i \\
i\end{array}$ & $\begin{array}{l}2 \\
+1 \\
\infty \\
n \\
0 \\
1\end{array}$ \\
\hline $\begin{array}{cc}* & 0 \\
= & \frac{3}{3} \\
\underline{E}\end{array}$ & & 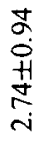 & $\begin{array}{l}\stackrel{0}{\dot{H}} \\
\text { 草 } \\
\dot{\sim}\end{array}$ & $\begin{array}{l}\underset{\dot{H}}{H} \\
\underset{\infty}{\stackrel{N}{N}}\end{array}$ & 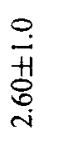 & $\begin{array}{l}\stackrel{2}{0} \\
\stackrel{0}{+} \\
\frac{1}{0} \\
i \\
i\end{array}$ & $\begin{array}{l}0 \\
\dot{0} \\
+ \\
\tilde{n} \\
n \\
0 \\
i\end{array}$ & $\frac{\vec{\sigma}}{\stackrel{+}{r}}$ & $\begin{array}{l}\infty \\
n \\
n \\
+1 \\
n \\
0 \\
i\end{array}$ & $\begin{array}{l}\infty \\
\infty \\
0 \\
+1 \\
2 \\
ٌ\end{array}$ & 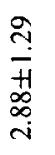 & 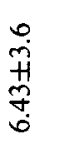 & \begin{tabular}{l}
\multirow{n}{n}{} \\
+ \\
$\tilde{n}$ \\
$\hat{n}$
\end{tabular} & $\begin{array}{l}\vec{y} \\
+ \\
m \\
\vec{m}\end{array}$ \\
\hline$B \stackrel{n}{g}$ & & $\begin{array}{l}\hat{a} \\
\dot{0}\end{array}$ & $=\infty$ & 苗 & 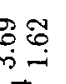 & $\begin{array}{l}0 \\
\dot{0} \\
+1\end{array}$ & \begin{tabular}{c} 
: \\
\hdashline \\
\hdashline
\end{tabular} & 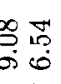 & $\begin{array}{ll}0 \\
6 \\
010\end{array}$ & $\stackrel{\infty}{\sim}$ & & 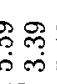 & 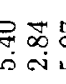 & 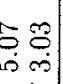 \\
\hline & & 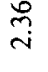 & $\stackrel{\infty}{i}$ & $\underset{i}{8}$ & $\underset{i}{\mathbb{J}}$ & $\frac{\infty}{\infty}$ & $\stackrel{2}{\circ}$ & 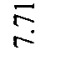 & $\begin{array}{l}80 \\
\infty \\
\infty\end{array}$ & $\approx$ & $\begin{array}{l}\vec{\circ} \\
\dot{\sigma}\end{array}$ & $\stackrel{\sim}{\tilde{r}}$ & $\overline{\mathrm{m}}$ & $\underset{\tilde{m}}{\sigma}$ \\
\hline $\bar{\circ}$ & $\frac{n}{\tilde{n}}$ & $\frac{\sigma}{I}$ & $L$ & $z$ & $\ll$ & $\stackrel{\mathscr{2}}{\Xi}$ & 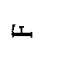 & $z$ & $\ll$ & $\vdash$ & $\stackrel{2}{1}$ & L. & $z$ & $<$ \\
\hline$\vec{I}$ & $\frac{0}{0}$ & 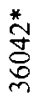 & & & & \begin{tabular}{l}
$\frac{*}{\infty}$ \\
$\infty$ \\
$\infty$ \\
\hdashline \\
$\varrho$
\end{tabular} & & & & & $\begin{array}{l}\stackrel{*}{5} \\
\stackrel{D}{\Xi} \\
\equiv\end{array}$ & & & \\
\hline
\end{tabular}


Table 2. continued

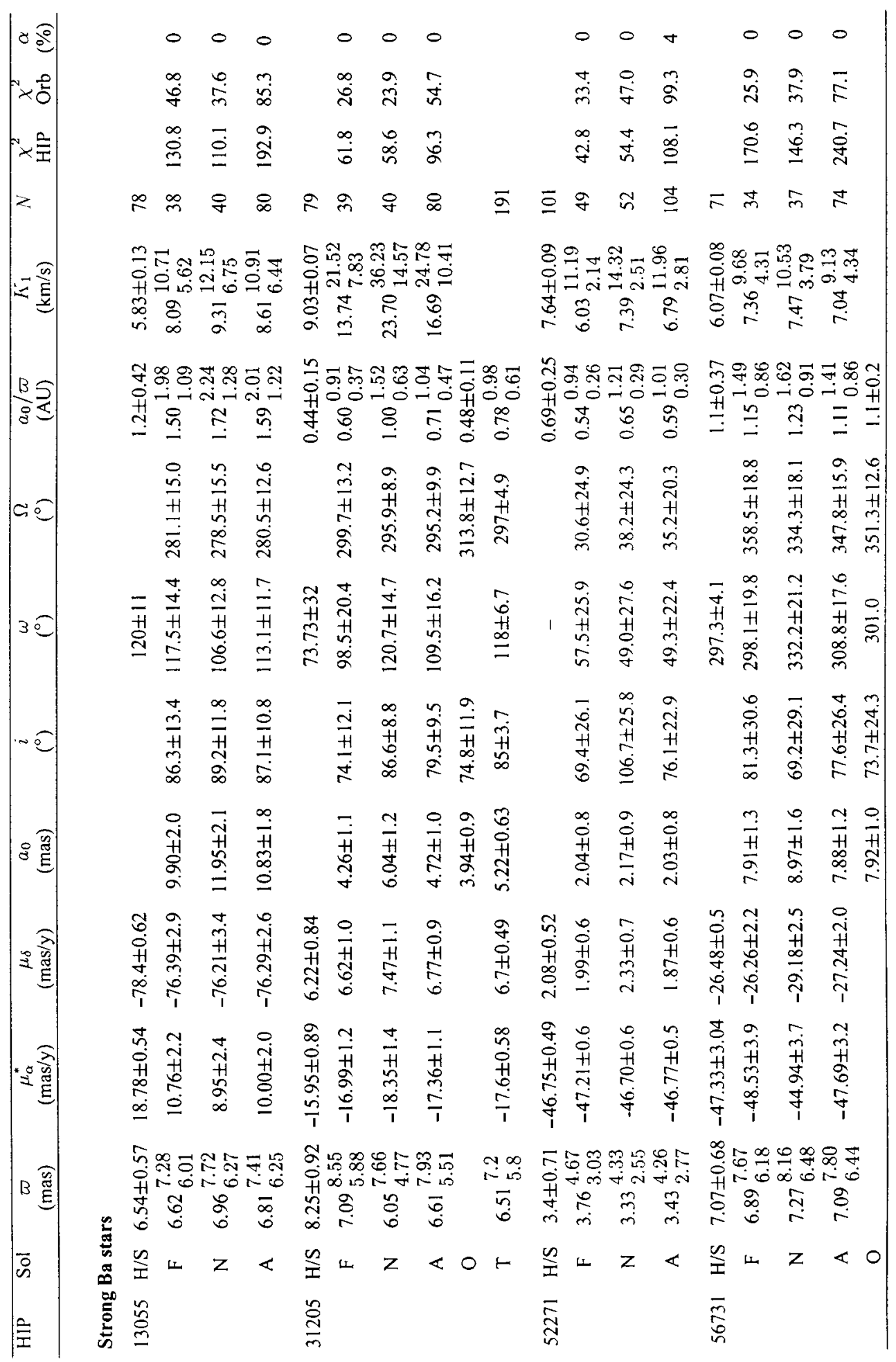


Table 2. continued

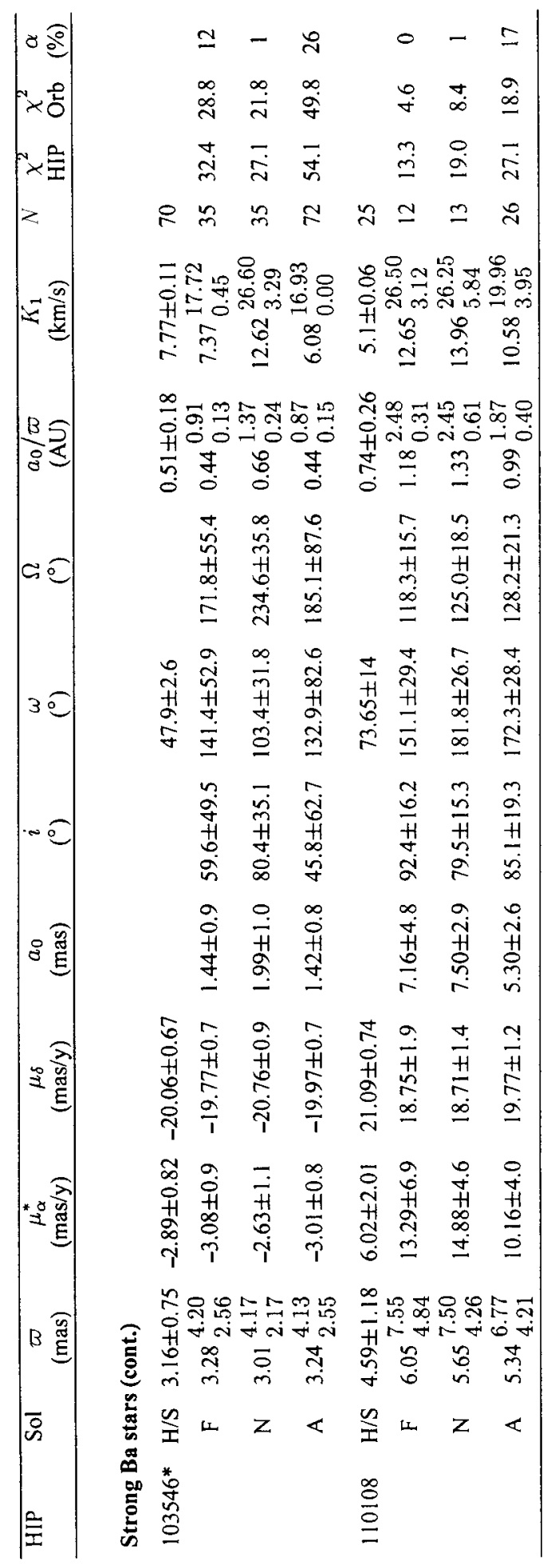


Table 2. continued

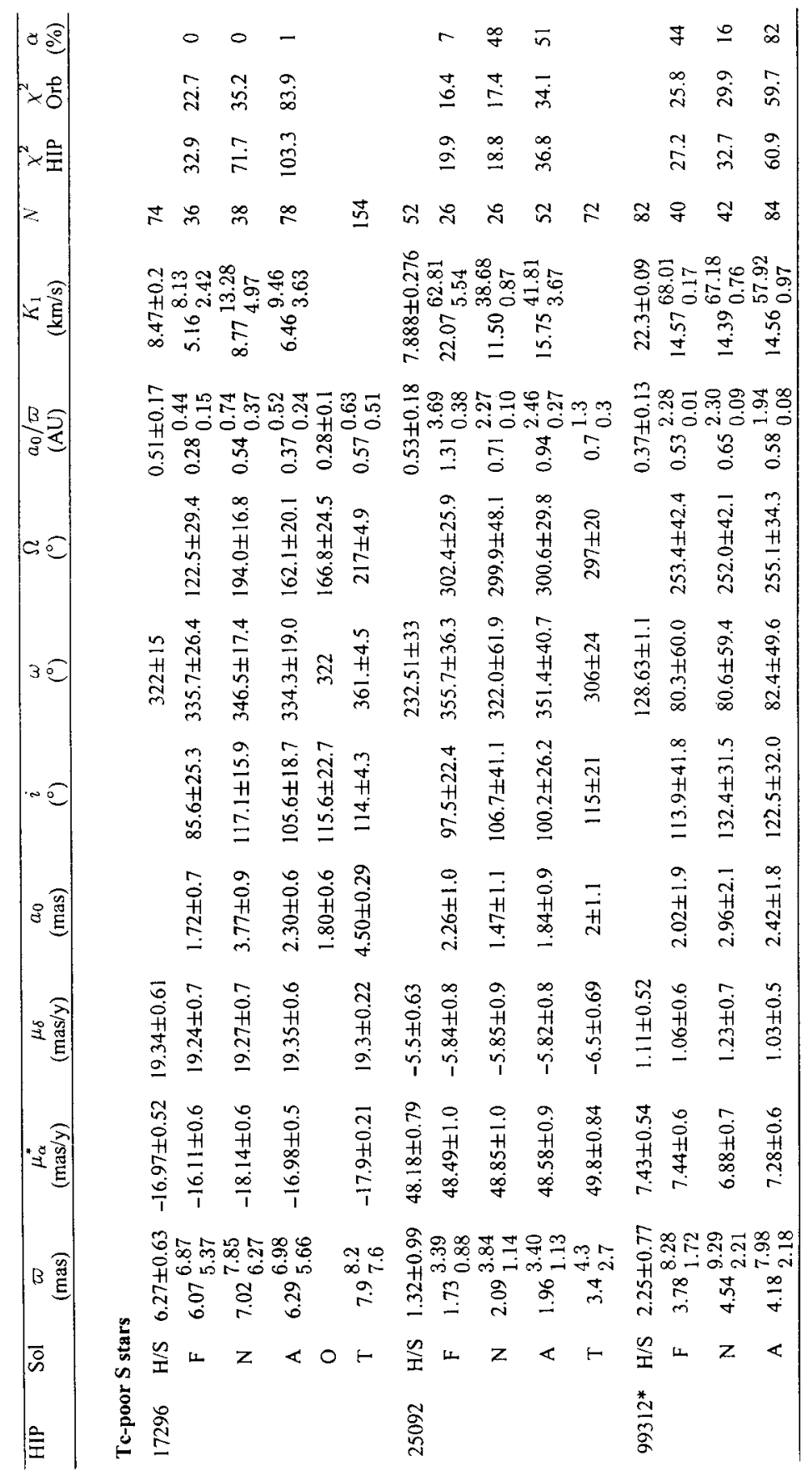

Remarks:

HIP 62409: only NDAC yields a realistic orbit.

HIP 116233, HIP 118266: orbits not well defined.

HIP 53763: orbit not well defined with large uncertainty on $\varpi$ since $P$ is close to 1 yr.

HIP 36042: large discrepancy between astrometric and astrophysical $a_{0} / \varpi$. However, the orbit has been retained since the correlation between the 9 parameters is very low (the average efficiency of the three solutions is 0.69 ; see Eq. (17)).

HIP 105881: orbit not well defined.

HIP 117607: although the orbit is not well defined, it has been kept in the Table to illustrate the correlations that may appear between $a_{0}$ and $\mu$.

HIP 103546: orbit not well defined.

HIP 99312: orbit not well defined. 

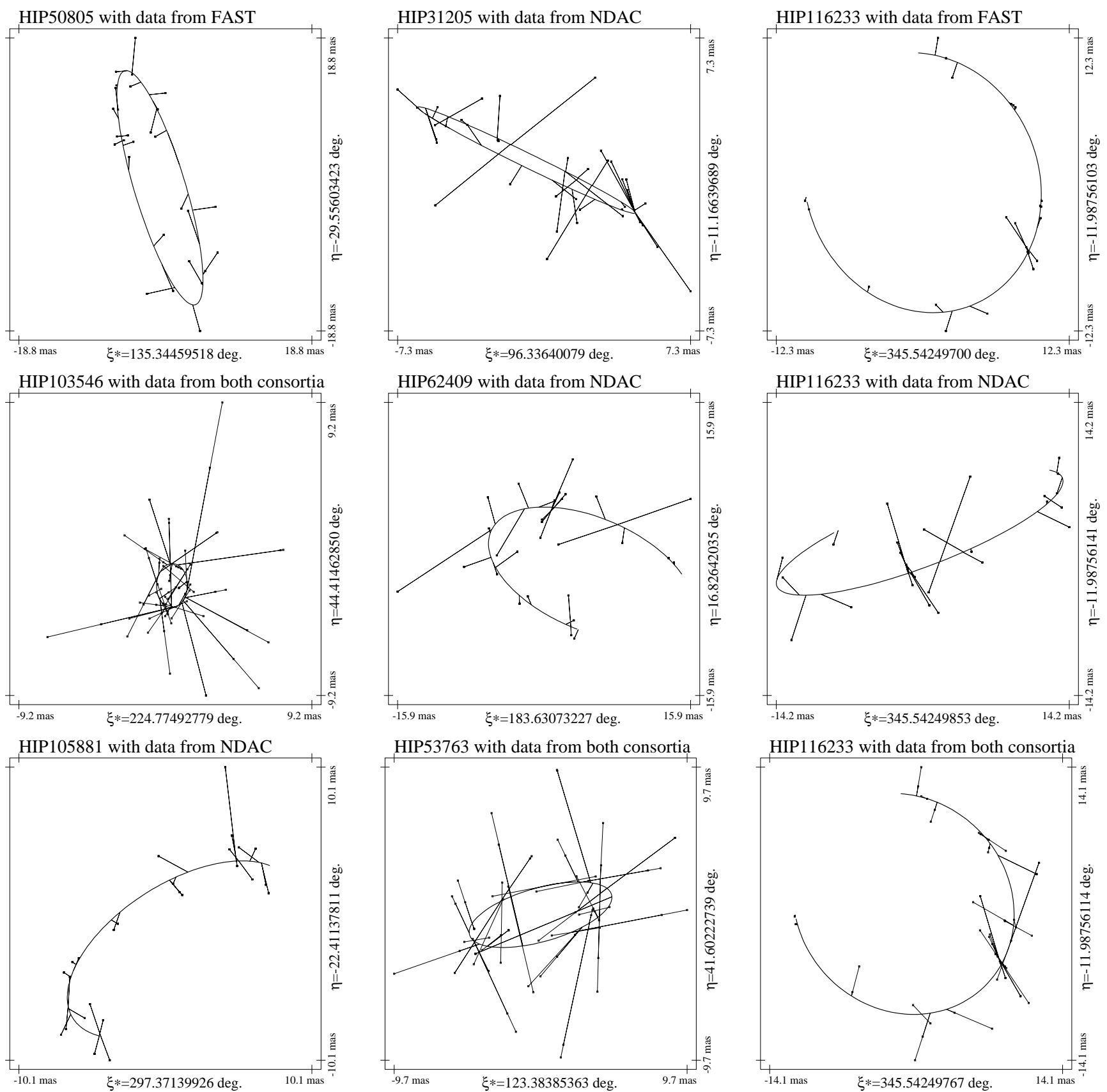

Fig. 1. The orbital arc on the plane tangent to the line of sight for some representative orbits among those listed in Table 2 . The segments connect the computed position on the orbit to the great circle (not represented, perpendicular to the segment) corresponding to the observed position (Hipparcos measurements are one-dimensional). HIP 50805 is the only system for which the DMSA/O provides an orbital solution from scratch; HIP 53763 has a small parallax (about 2 mas), not very well determined since the orbital period is close to $1 \mathrm{yr}$; only the NDAC solution is acceptable for HIP 62409; the orbit of HIP 103546 is at limit of what can be extracted from the IAD; HIP 105881 is an example of an incomplete, albeit well determined, orbital arc; HIP 116233 is one case where the NDAC and FAST solutions are rather different 
parallaxes...) have instead been selected and are presented in Fig. 1.

\subsection{Comparison with DMSA/O solutions}

For HIP 17296 (Tc-poor S), 31205 (strong Ba) and 56731 (strong Ba), orbital solutions are provided in the DMSA/O and were derived using spectroscopic elements from the literature. HIP 50805 (dwarf Ba) is the only case in our sample where an orbit could be derived from scratch by the Hipparcos consortia. For all these systems, the astrometric orbits derived by the methods described in Sect. 2 are in excellent agreement with the DMSA/O elements, thus providing an independent check of the validity of our procedures. Further checks are presented in Sect. 3.2.

The large number (23) of systems for which orbital solutions could be extracted from the Hipparcos data (as compared to only 4 of those already present in the DMSA/O) illustrates the great potential that still resides in the Hipparcos IAD or TD.

\subsection{Check of the astrometric orbit}

Several checks are possible to evaluate the accuracy of the astrometric elements derived in the present paper.

First, it is possible to compare the astrometric and spectroscopic values of $\omega$, the argument of periastron. In most cases, the two determinations agree within $2 \sigma$ (Fig. 2). However, even when the orbital period is shorter than the Hipparcos mission, the $\omega$ derived from the IAD is seldom as precise as the spectroscopic one.

In a few cases, the spectroscopic orbit is assumed to be circular. In that case, the time $T$ of passage at periastron becomes meaningless, and is replaced by the time of the nodal passage (or, equivalently, the time of maximum radial velocity). This is equivalent to setting $\omega$ equal to 0 . Non-zero values for $\omega$ would correspond to other conventions for the origin epoch. Among our systems with acceptable orbits, two have circular orbits: HIP $53763(\mathrm{CH}$ star) and HIP 52271 (strong barium star). For these systems, our fit leads to values of $\omega$ significantly different $(2 \sigma)$ from 0 , indicating that at the reference epoch, the star is in fact far from the node where it was expected to be.

It is also possible to compare the astrometric value of $K_{1}$ (using in Eq. (12) the value of $a, i$ and $\varpi$ from the astrometry and $e$ and $P$ from the spectroscopy) with the spectroscopic value. Figure 3 shows that, even if $a_{0} / \varpi$ is well defined, the inclinations are generally not very accurately determined, thus leading to uncertain values of $K_{1}$. This unfortunate property of $i$ is well illustrated in Figs. 4 and 5. One example where the accuracy of the astrometric value of $i$ must be questioned is the dwarf barium star HIP 105969: despite the fact that the astrometric $a_{0} / \varpi$ ratio perfectly agrees with its estimate based on the masses,

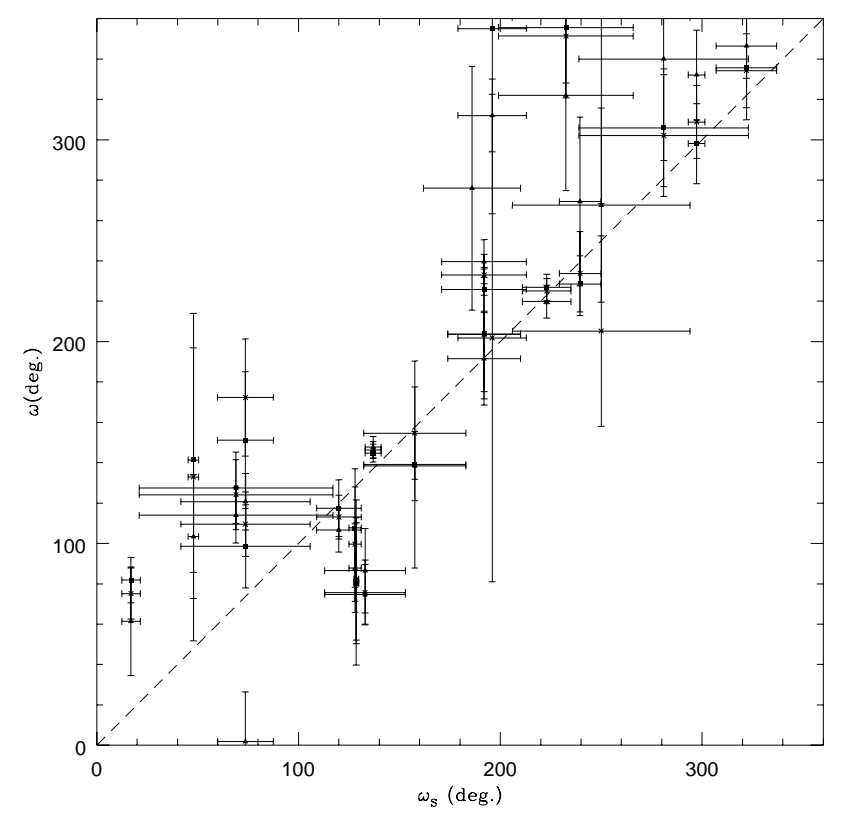

Fig. 2. Comparison of the astrometric (ordinate) and spectroscopic (abscissa) determinations of the argument of periastron $\omega$

the astrometric prediction of the semi-amplitude of the radial-velocity variations differs by almost of factor of 2 as compared to the actual spectroscopic value (Table 2). The only way to resolve that discrepancy is to assume that the orbital inclination is largely in error.

The semi-major axis $R=a_{0} / \varpi$ as derived from its astrophysical estimate (Eq. 13) is compared to its astrometric value in Fig. 6, and the two values are often consistent with each other. Although the value of $a_{0}$ is likely to be affected by a positive bias (i.e., a positive $a_{0}$ value is derived even when the data consist of pure noise, as clearly apparent from the astrometric $a_{0} / \varpi$ values listed in Table 1 ), this bias does not markedly affects the retained solutions displayed in Fig. 6, except for solutions with $\varpi<3$ mas, which all have $R / \hat{R}>1$. Solutions for larger $\varpi$ values are almost equally distributed around unity.

\subsection{Comparison TD vs. IAD}

Among the 81 systems studied in this paper, 22 have TD and only 5 belong to the list of accepted orbits (HIP 17296 , $25092,31205,5080$, and 105881). They hence can be used to check the consistency of the two orbit-determination methods. The astrometric orbits derived from the TD are given in Table 2 as lines labeled with "T" in Col. 2. The agreement between the results obtained with the two data types is quite good (within $1 \sigma$ error bars). The number of TD measurements for those five systems is up to twice as large the number of IAD measurements. As a consequence, the confidence intervals of the parameters is systematically narrower. One should however keep in mind 


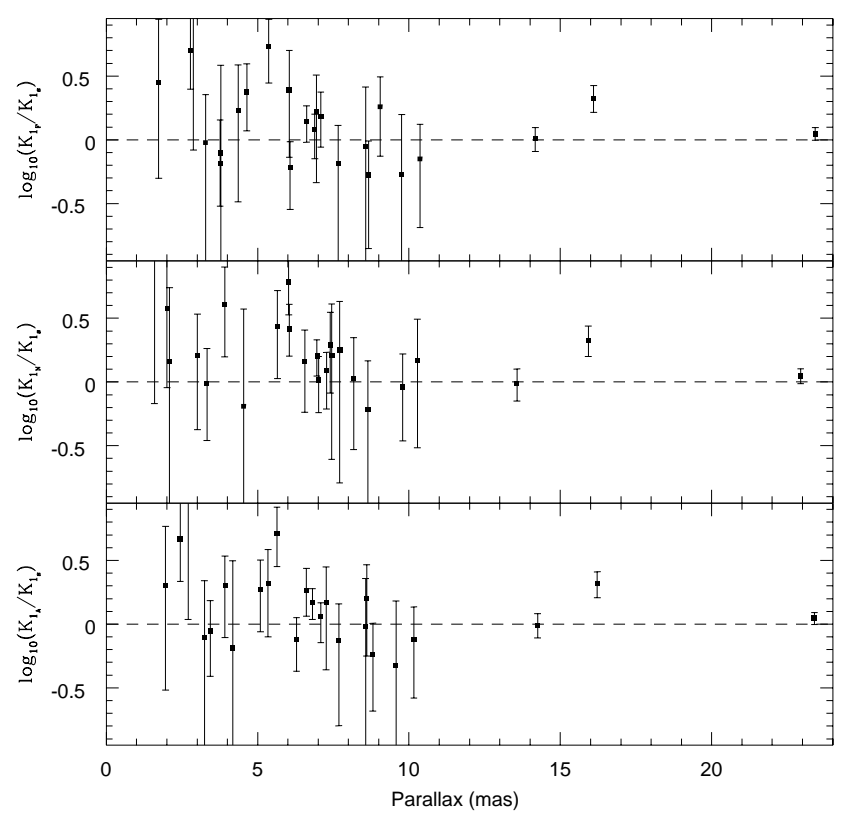

Fig. 3. Comparison of the semi-amplitude of the radial velocity curve derived from the astrometry (using Eq. 12) and the spectroscopic value. The three panels show the results derived from FAST, NDAC and FAST+NDAC (from top to bottom respectively)

that more precise does not imply more accurate. For instance, in the case of the mild Ba star HIP 105881, the 86 TD measurements yield $a_{0} / \varpi=1.59$ (with $1 \sigma$ limits of 1.45 and 1.74) as compared to $1.2 \pm 0.35$ estimated from Kepler's third law. The 54 IAD measurements from FAST and NDAC combined yield a more accurate result of 0.99 (between 0.44 and 1.69).

For the sake of completeness, we checked the astrophysical consistency of the orbit derived for the 22 systems for which TD are available. All systems but the five already accepted were rejected. In essence, this confirms that the astrometric content of the TD is basically equivalent to the IAD and that nothing new can come out from TD if IAD do not yield a reliable solution.

\section{Comparison of parallaxes and proper motions derived from single- or double-star solutions}

It has been stressed (Wielen 1997; Wielen et al. 1999) that the proper motions provided by the Hipparcos catalogue may be systematically in error for long-period binaries (i.e. with $P>3 \mathrm{yr}$ ), if those were not recognized as such by the reduction consortia. The orbital motion may then add to the actual proper motion, changing both its direction and modulus. The present sample offers a good opportunity to evaluate the impact of this effect.

Figures 7 and 8 compare the position angle and modulus, respectively, of the Hipparcos proper motion with

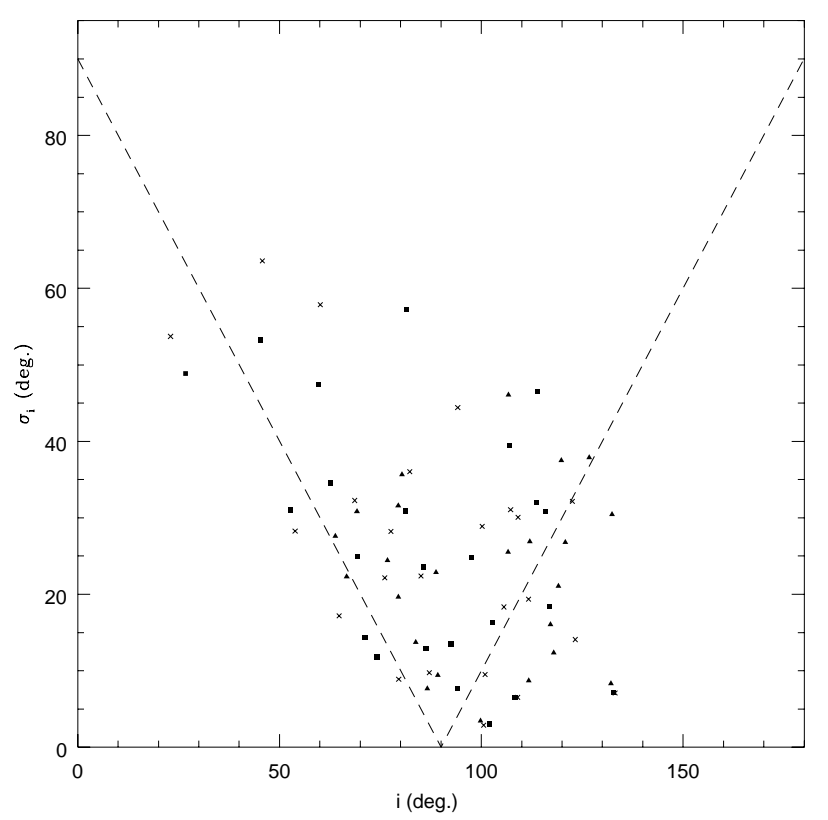

Fig. 4. Uncertainty on the inclination $i$ as a function of $i$. All points lying inside the region delineated by the oblique dashed lines have orbital inclinations consistent with $i=90^{\circ}$. Filled triangles correspond to orbital solutions derived from NDAC, filled squares to FAST and crosses to NDAC+FAST

those derived when account is made of the orbital motion as in the present work. It is clearly apparent that the direction of the proper motion listed in the Hipparcos catalogue is correct, as expected, for orbits with periods less than $3 \mathrm{yr}$, corresponding to the duration of the Hipparcos mission. The Hipparcos values are the less accurate in the orbital-period range 3 to about 5 yr. The position angle quoted by the Hipparcos catalogue may then be off by several dozens degrees (a good illustration of this situation is offered by the the strong Ba star HIP 110108 in Table 2), though it differs generally by less than $3 \sigma$ from the correct value. For longer orbital periods, the orbital motion becomes negligible over the duration of the Hipparcos mission, and the proper motions are again rather well determined in the Hipparcos catalogue. The situation is almost identical for the the proper-motion modulus, except that the error bars now become very large for orbital periods longer than $3 \mathrm{yr}$. This situation translates the fact that the proper motion and the semi-major axis are strongly correlated (resulting in a large formal uncertainty on the proper motion), because the two motions are difficult to disentangle when the Hipparcos data sampled only a fraction of the orbit. This situation is encountered e.g. for HIP 104785 and 104732 (whose astrometric orbits have not been retained precisely because of the large uncertainty on $a_{0}$ introduced by the strong correlation with $\mu$ ), and results in sometime large differences between the $a_{0}$ and $\mu$ values derived from the NDAC, FAST and NDAC+FAST data 


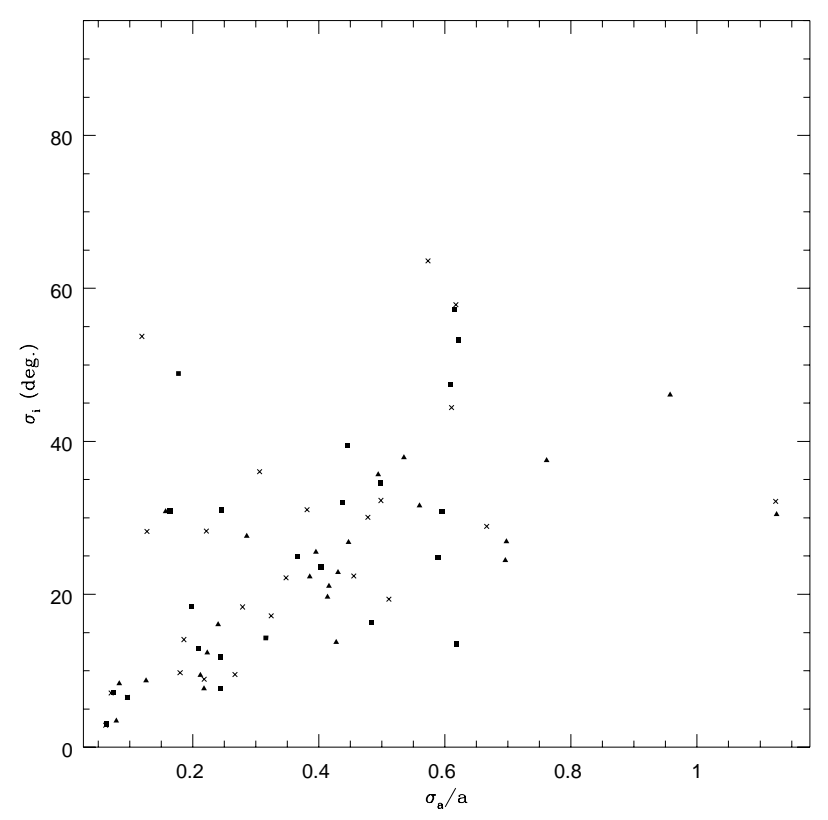

Fig. 5. Correlation between the uncertainties on the inclination $i$ and the semi-major axis $a_{0}$. The symbols have the same meaning as in Fig. 4

sets, since they are now very sensitive to the measurement errors.

Figure 9 compares the parallax listed in the Hipparcos catalogue with that derived in the present work, as a function of the orbital period. It turns out that the agreement is good, except for systems with orbital periods close to 1 yr. For those systems, the parallax cannot be accurately derived since the orbital motion and the parallactic motion are strongly entangled. This situation is encountered for HIP 17402, 53763 and 62827. Large error bars at periods different from 1 yr correspond to systems with parallaxes smaller than 3 mas.

A few stars (HIP 8876, 29740, 29099, 32831, 36613) in our sample had negative parallaxes in the Hipparcos catalogue. The parallaxes we derive for these systems are slightly positive ( $\varpi^{\prime}$ was adopted to guarantee that property), but the associated error bar encompasses zero, so that these new parallaxes are just useless.

\section{Masses}

The test presented in Sect. 2.1.5 (Eq. 13) to evaluate the astrophysical relevance of the orbital solution on the basis of $a_{0} / \varpi$ is in essence based on an a priori knowledge of the masses, since Eq. (13) may in fact be rewritten as $Q=\frac{M_{2}^{3}}{\left(M_{1}+M_{2}\right)^{2}}=\frac{\left(a_{0} / \varpi\right)^{3}}{P^{2}}$.

The orbital solutions that appear to be statistically significant on the basis of the F-test in almost all cases turned

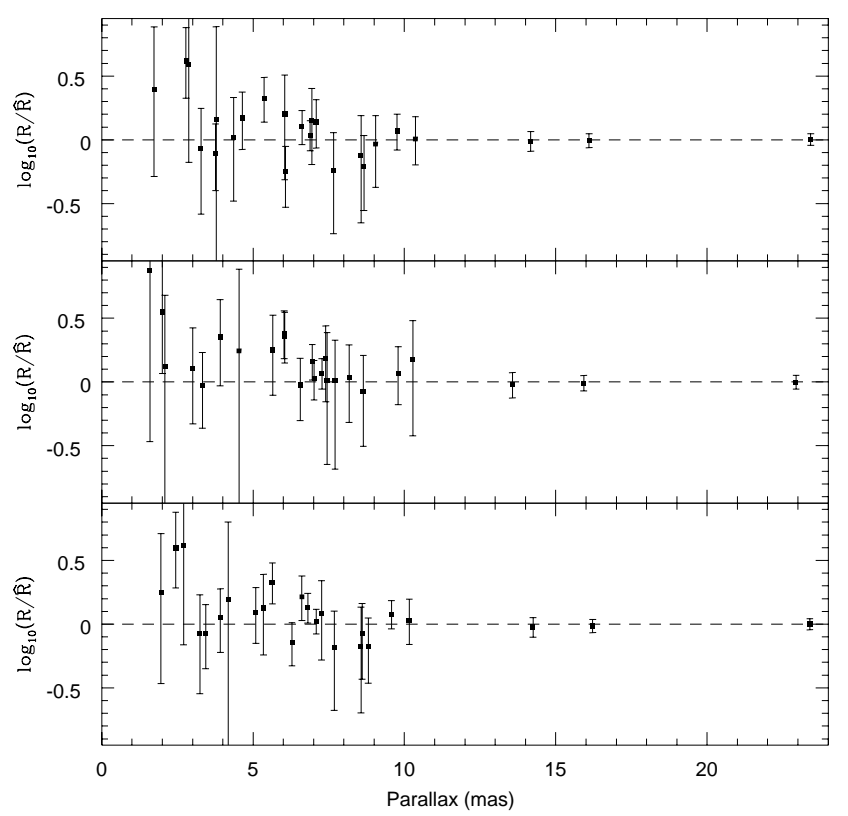

Fig. 6. Comparison of the semi-major axis $R=a_{0} / \varpi$ derived from the astrometry and from Eq. (13). The upper panel displays the results from the FAST data, the middle panel the results from the NDAC data, and the lower panel the results from FAST and NDAC combined. The error bars assume that $M_{1}, M_{2}$ and $P$ are error-free

out to have a semi-major axis $a_{0} / \varpi$ consistent with its expected value based on the estimated masses, the dwarf barium star HIP 60299 being however a notable exception. This agreement is well illustrated in Fig. 6.

Therefore, the present analysis does not add much to our previous knowledge of the masses, especially since the astrometric orbit only allows to eliminate $i$ from the mass function but does not give access to the individual masses.

It was originally hoped that the present astrometric results might be used to test the hypothesis which played a central role in the statistical analysis of the mass functions of CPRS (Jorissen et al. 1998), namely that their $Q$ distributions are quite peaked, because they host a white dwarf companion. However, the error bar on $a_{0} / \varpi$ (and hence on $Q$ ) is too large, even for the best determined bariumdwarf orbits (Table 2), to be able to draw a meaningful $Q$-distribution from the astrometric orbits.

\section{Conclusions}

The availability of the Hipparcos Intermediate Astrometric Data and, when they exist, the Transit Data, allow to improve upon the existing Hipparcos catalogue. Once a new or revised spectroscopic binary orbit becomes available, it can be used to update the Hipparcos astrometric parameters and, sometime, to derive the corresponding astrometric orbit. In the latter 


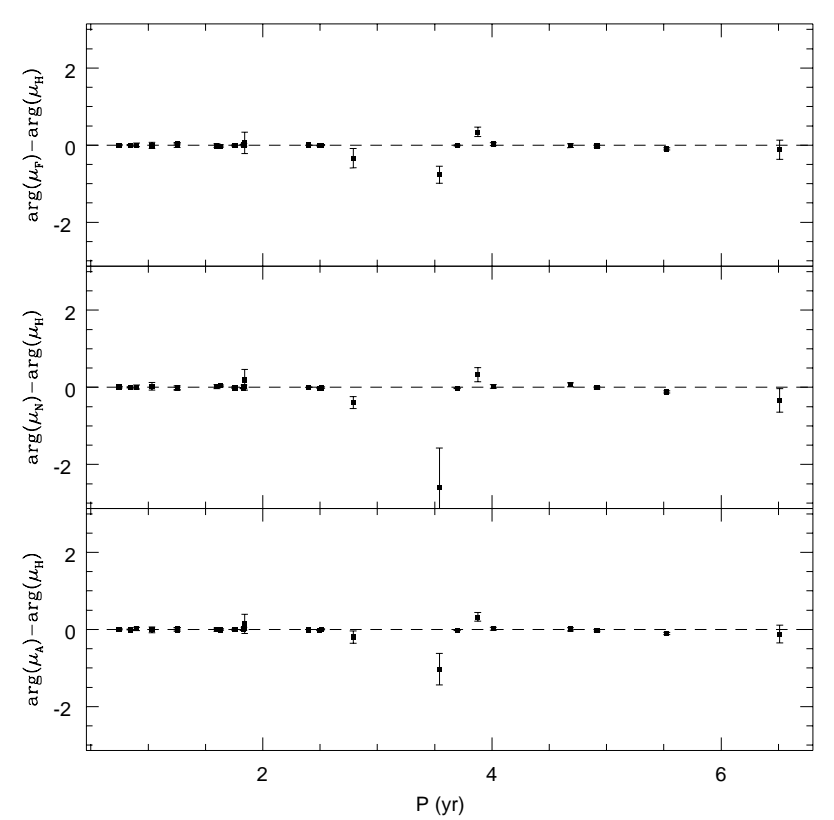

Fig. 7. Comparison of the position angle (expressed in radians) of the Hipparcos proper motion with that derived when account is made of the orbital motion (present work), as a function of the orbital period. The upper panel displays the results from the FAST data, the middle panel the results from the NDAC data, and the lower panel the results from FAST and NDAC combined. Error bars do not include the uncertainty on the Hipparcos values

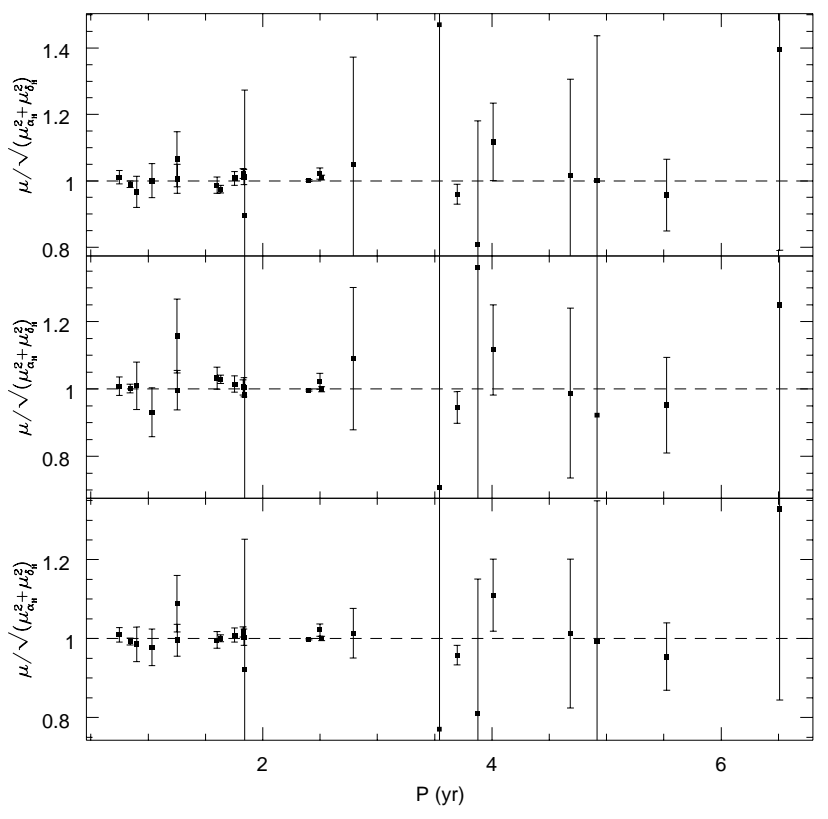

Fig. 8. Same as Fig. 7 for the proper-motion modulus

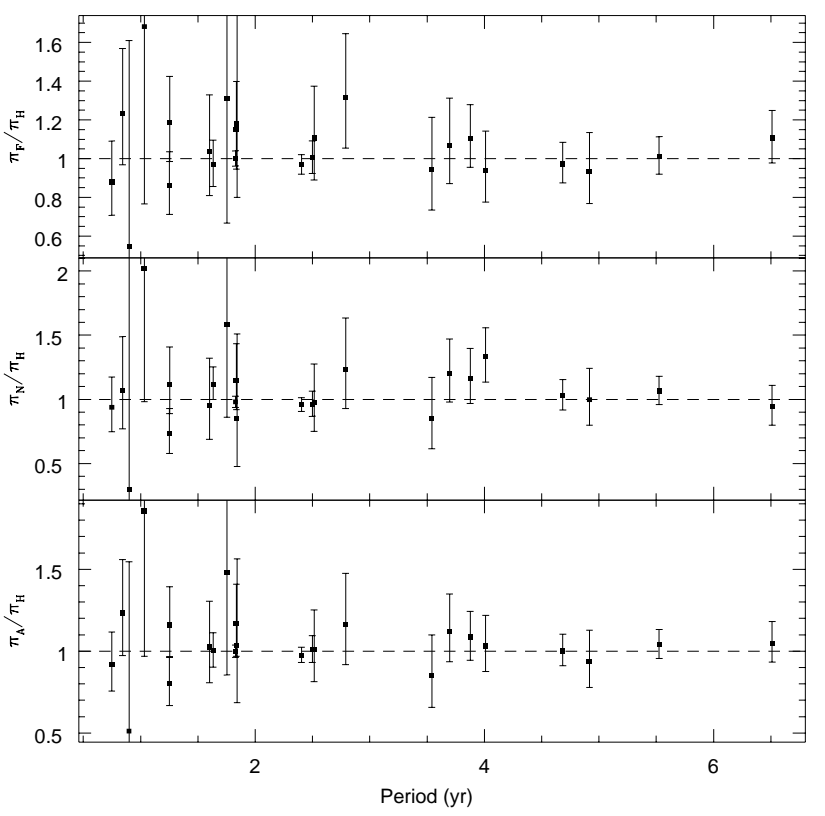

Fig. 9. Comparison of the Hipparcos parallax with that derived when account is made of the orbital motion (present work), as a function of the orbital period. As in Fig. 7, results from FAST, NDAC and FAST+NDAC are presented in the upper, middle and lower panels, respectively. Error bars include only the uncertainty on the parallaxes derived from the present work, and are computed according to Eq. (9)

case, the orbital inclination is obtained, thus allowing to improve our knowledge of the binary system.

The optimization method used in the present paper yields a solution in all cases, and it is therefore important to perform consistency checks. One test which may be applied in all cases involves the comparison of the arguments of the periastron and of the semi-amplitude of the radial-velocity curve, as derived from either the spectroscopic orbit or the astrometric one. The comparison of the semi-amplitude of the radial-velocity curve involves however the inclination of the orbit which is not always very well determined.

In this context, chemically-peculiar red stars (CPRS) like barium, Tc-poor $\mathrm{S}$ and $\mathrm{CH}$ stars are interesting because they provide their own consistency checks. Indeed, beside the orbit which can be determined as for any other spectroscopic binary, the mass of both components may be guessed with some confidence. These mass estimates based on astrophysical considerations may then be compared to the mass of the system derived from the astrometric orbit using Kepler's third law.

From a sample of 81 CPRS spectroscopic systems whose orbits became available after completion of the Hipparcos catalogue, we have derived 23 reliable astrometric orbits. This shows that the number of Hipparcos entries for which an orbital solution may be obtained is 
much larger than suggested by the number of existing entries in the DMSA/O.

Updated astrometric parameters from this particular sample have shown that the Hipparcos-catalogue parallaxes are not reliable for systems with orbital periods close to 1 yr. Similarly, the Hipparcos proper motions are not reliable for unrecognized binaries with periods in the range 3 to about $8 \mathrm{yr}$, as already suspected by Wielen et al. (1999).

The orbit-determination methods based on the IAD and TD being completely different, systems for which the two sets of data are available can be used to assess the consistency of the solutions derived from both sets with different methods. It turns out that the two sets of results are generally in good agreement.

Acknowledgements. We thank P. North for communicating us the orbital elements of dwarf $\mathrm{Ba}$ stars in advance of publication. We also thank two anonymous referees for their very constructive comments.

\section{References}

Bergeat J., Knapik A., 1997, A\&A 321, L9

Bevington P.R., Robinson D.K., 1992, Data reduction and error analysis for the physical sciences. McGraw-Hill, 2nd edition

Binnendijk L., 1960, Properties of Double Stars. University of Pennsylvania Press

Boffin H.M.J., Cerf N., Paulus G., 1993, A\&A 271, 125

Dennis Jr. J.E., Schnabel R.B., 1995, Numerical Methods for Unconstrained Optimization and Nonlinear Equations, SIAM, 2nd edition

Eichhorn H., 1989, Bull. Astron. Inst. Czechosl. 40 (6), 394
ESA, 1997, The Hipparcos and Tycho Catalogues, ESA SP1200

Girard T.M., Wu H., Lee J.T., et al., 2000, AJ 119, 2428

Heintz W.D., 1978, Double stars. D. Reidel Publishing Company

Jorissen A., Van Eck S., Mayor M., Udry S., 1998, A\&A 332, 877

Lucy L.B., Sweeney M.A., 1971, AJ 76, 544

Marquardt D.W., 1963, SIAM J. 11, 431

McClure R.D., 1983, ApJ 268, 264

McClure R.D., 1997, PASP 109, 536

McClure R.D., Woodsworth A.W., 1990, ApJ 352, 709

Mennessier M.O., Luri X., Figueras F., et al., 1997, A\&A 326, 722

Mood A.M., Gaybrill F.A., Boes D.C., 1974, Introduction to the Theory of Statistics. McGraw-Hill, 3 edition

North P., Jorissen A., Mayor M., 1999, in Wing R. (ed.), The Carbon Star Phenomenon, IAU Symp. 177. Kluwer Academic Publishers

Pourbaix D., 1994, A\&A 290, 682

Pourbaix D., 1998a, in De Leone R., Murli A., Toraldo G., Pardalos P.M. (eds.), High Performance Software for Nonlinear Optimization: Status \& Perspective. Kluwer Academic Publishers

Pourbaix D., 1998b, A\&AS 131, 377

Pourbaix D., Eichhorn H., 1999, A\&AS 136, 419

Quist C.F., Lindegren L., 1999, A\&AS 138, 327

Van Eck S., Jorissen A., Udry S., Mayor M., Pernier B., 1998, A\&A 329, 971

van Leeuwen F., Evans D.W., 1998, A\&AS 130, 157

Wielen R., 1997, A\&A 325, 367

Wielen R., Schwan H., Dettbarn C., Jahreiss H., Lenhardt H., 1997, in ESA (ed.), Hipparcos - Venice '97, ESA SP-402, p. 727

Wielen R., Dettbarn C., Jahreiß H., Lenhardt H., Schwan H., 1999, A\&A 346, 675 